CIRJE-F-959

\title{
Deflation/Inflation Dynamics: Analysis based on Micro Prices
}

\author{
Hiroshi Yoshikawa \\ The University of Tokyo \\ Hideaki Aoyama \\ Kyoto University and RIETI \\ Yoshi Fujiwara \\ The University of Hyogo \\ Hiroshi Iyetomi \\ Niigata University \\ February 2015
}

CIRJE Discussion Papers can be downloaded without charge from:

http://www.cirje.e.u-tokyo.ac.jp/research/03research02dp.html

Discussion Papers are a series of manuscripts in their draft form. They are not intended for circulation or distribution except as indicated by the author. For that reason Discussion Papers may not be reproduced or distributed without the written consent of the author. 


\title{
Deflation/Inflation Dynamics: Analysis based on Micro Prices
}

\author{
Hiroshi Yoshikawa ${ }^{1,5 *}$, Hideaki Aoyama ${ }^{2,5}$, \\ YOSHI FuJIWARA ${ }^{3}$, AND Hiroshi IYetomi ${ }^{4}$ \\ ${ }^{1}$ Graduate School of Economics, The University of Tokyo, Tokyo 113-0033, Japan \\ ${ }^{2}$ Graduate School of Sciences, Kyoto University, Kyoto 606-8502, Japan \\ ${ }^{3}$ Graduate School of Simulation Studies, The University of Hyogo, Kobe 650-0047, Japan \\ ${ }^{4}$ Department of Mathematics, Niigata University, Niigata 950-2181, Japan \\ ${ }^{5}$ Research Institute of Economy, Trade and Industry (RIETI), Tokyo 100-8901, Japan
}

February 16, 2015

\begin{abstract}
Micro price data shows that individual price settings are not time-invariant as presumed in the existing literature. Furthermore, the analysis of autocorrelations shows that interactions of micro prices with leads and lags ignored in the literature play a very important role in explaining the behavior of aggregate price index. The price index such as CPI contains "noises" for the purpose of macroeconomics and monetary policy. The "core" CPI used by central banks is, however, defined merely on common sense and casual observation. We present a new method of extracting information on the systemic changes of the aggregate price based on micro price data. The "true core price index" so defined is correlated with over-time hours worked, the unemployment rate, and the exchange rate. It is not significantly correlated with money supply. Our analysis also shows that inertia arising from interactions of micro prices more plausibly explains the behavior of aggregate price than expectations.
\end{abstract}

Key words: CPI, sticky prices, Hilbert transformation, CPCA

JEL classification: E31, D12, C40

*Corresponding author, Email: yoshikawa@e.u-tokyo.ac.jp 


\section{Introduction}

How prices behave is of primary importance in economics. In macroeconomics, inflation, together with unemployment, is one of the most important policy issues. More recently, deflation is regarded as a threat to the macroeconomy. Many central banks are indeed committed to explicit inflation target such as the annual two percent increase of consumer price index (CPI). Facing the zero interest bound, they struggle against deflation by resorting to quantitative easing (QE). The efficacy of such policy depends, of course, on how prices are determined.

In macroeconomic theory, prices are said to be "sticky". In fact, in the modern DSGE (dynamic stochastic general equilibrium) models, monetary policy is effective to the extent that prices are sticky. There are a number of theories which attempt to explain sticky prices: the Taylor-Calvo model of desynchronized staggered wage/price changes (Calvo, 1983) and menu cost models (Mankiw, 1985), just to name a few. Based on such micro-foundations, the standard framework for understanding the role of monetary policy is the New Keynesian Phillips curve (NKPC).

The key property of the NKPC is that inflation is primarily a forward-looking process. That is, expectations on future inflation largely determine current inflation. This justifies recent emphasis on expectations management and communications as tools of monetary policy. There is a great amount of literature on the NKPC. However, after a long survey of the literature, Mavroeidis et al. (2014) reached quite disappointing conclusion. Namely, their major finding is that estimation of the NKPC using macro data is subject to a severe weak instruments problem. Indeed, they find that "the evidence is consistent both with the view that expectations matter a lot, as well as with the opposite view that they matter very little". They thus conclude that identification of the NKPC is too weak to warrant research on conceptually minor extensions. The traditional analysis based on macro data has its clear limitations.

Meanwhile, recent empirical works on micro price-setting as surveyed by Klenow and Malin (2011) have uncovered hitherto little known dynamics of micro prices. Bils and Klenow (2004), for example, by examining the frequency of price changes for 350 categories of goods and services demonstrate that half of prices last 5.5 months or less. Their findings seem to suggest that individual prices are actually not rigid. There are substantial differences across goods, however; prices of raw materials and foodstuff are flexible while those of services less flexible. The fact is well known. Thus, central banks are committed to inflation targeting with respect to the "core" CPI which excludes prices of foodstuff and energy.

Studies of micro prices provide useful information. However, changes of aggregate price index are entirely different matter from changes of individual prices. For example, micro price changes include temporary sales. The recent literature discusses whether or not temporary sales should be taken into account for the purpose of exploring price rigidity in macroeconomics. Some such as Nakamura and Steinsson (2013) take it simply that "a price change is a price change, i.e., that all price changes inclusive of temporary sales should be counted equally." However, plainly 
temporary sales are not a kind of price change we are interested in for the purpose of macroeconomics and monetary policy. In any case, the recent studies demonstrate that the regular price exclusive of temporary sales continues to be the dominant factor in determining the time trajectory of the aggregate price level (Midrigan, 2011). For this reason, we analyze the regular price exclusive of temporary sales in the present paper.

For changes of aggregate price index such as CPI, the frequency of individual price changes and synchronization on which many empirical works focus provides only partial information. The reason is that deflation and inflation are nothing but changes in the aggregate price over time while the existing literature on micro prices focuses mostly on cross-sectional differences among micro prices. There still remains much to be done.

The major purpose of this paper is twofold. First, the existing literature on micro price dynamics either explicitly or implicitly assumes that frequency and synchronization of micro price changes are independent of each other and are time-invariant. More generally, probability distribution of micro price changes is assumed to be given and time-invariant; alternative theories are proposed to account for such a given distribution (Golosov and Lucas (2007), Midrigan (2011)). However, distribution of micro price changes is actually not time-invariant. Moreover, prices of individual goods and services affect each other with leads and lags. In Section III, we formally demonstrate this fact by way of the analysis of autocorrelations of prices. Therefore, it is essential to analyze dynamics of micro prices taking explicitly account of these lead and lag relationships. The present paper precisely does it. The analysis sheds light on the central question for macroeconomics and monetary policy, namely the relative importance of expectations and inertia as determinant of aggregate price.

Secondly, individual prices occasionally change simultaneously responding to certain macro shocks. Despite of our primary interest in macroeconomics and monetary policy, the existing literature does not empirically link the findings on micro price behavior to changes in macroeconomic variables, particularly money supply which plays the central role in standard theoretical models. In some papers such as Golosov and Lucas (2007) and Midrigan (2011), money is explicitly introduced, but it is simply assumed that money supply must directly affect micro prices. Here, theory is ahead of hard empirical evidence. This is a pity because the analysis of micro price dynamics should be able to provide useful empirical information.

As discussed previously, estimation of the NKPC which directly relates actual inflation to macro variables has its clear limitations. In fact, actual deflation and inflation defined by the standard aggregate price indices contain "noises" for the purpose of macroeconomics and monetary policy. That is why central banks target at the "core" CPI rather than CPI itself; economists well recognize that prices of foodstuff can sizably change due to climate changes which for the purpose of monetary policy, we can reasonably take as "noises." The "core" inflation which plays such an important role for policy making is, however, defined merely on common sense and casual observation. Taking advantage of micro prices, we can extract information on the "systematic" changes of the aggregate price. Furthermore, once we obtain the 
"systematic" changes of the aggregate price, we can explore to what macro variables they are significantly related.

In section II] studying 830 prices of goods and services for Japan, we demonstrate that the frequency of individual price changes and synchronization are, in fact, not constant but time-varying. The existing literature routinely assumes that distribution of micro price changes is constant. However, this assumption is simply not borne out by data. Frequency, synchronization, and size of price changes are all time-varying. Moreover, they change in clusters, not simultaneously in the economy as a whole. In this respect, there is a significant gap between observed facts and theory because in standard theory, changes in money, supposedly the most important macro disturbance, affect more or less uniformly all the prices. In section III, we examine the autocorrelations of individual prices and the aggregate CPI. This analysis demonstrates the importance of interdependence of individual prices with leads and lags. Section IV analyzes such lead-lag dynamics of individual prices by a new method, and defines the "systemic" changes in the aggregate price. The aggregate price index so defined is a kind of "true core" price. Section $\mathrm{V}$ then explores what are the major macroeconomic variables which produce such "systemic" changes in the aggregate price. In standard macroeconomic model, money supply determines changes in the aggregate price in the long-run. It is a cliché that deflation/inflation is always "monetary phenomenon". However, it has not been explicitly analyzed whether money actually affects the aggregate price consistent with changes of individual prices. Section $\mathrm{V}$ also explores the relative importance of expectations and inertia as determinant of aggregate price. Section VI offers concluding remarks.

\section{Individual Prices}

We examine the Japanese monthly data of the following three categories of individual prices for the period from January 1980 to June 2013.

IPI: Import Price Index, compiled by the Bank of Japan (BoJ) consists of "prices of $\cdots$ imports at the stage of entry into Japan." It covers 75 goods (Bank of Japan, 2014).

DCGPI: Domestic Corporate Goods Price Index, compiled by the BoJ, "surveys the prices of goods traded among companies, specifically domestically produced goods for domestic markets, mainly at the stage of shipment from producers and partly from wholesalers." It covers 420 goods (Bank of Japan, 2014).

CPI: Consumer Price Index, compiled by the Statistics Bureau of the Ministry of Internal Affairs and Communications covers 335 consumption goods and services (Statistics Bureau, 2014).

Altogether, we have prices of 830 goods and services for the period from January 1980 to June 2013, namely, 402 months 11 They cover a wide range of goods and

\footnotetext{
${ }^{1}$ The number of goods and services of all three indices have been gradually increasing since 1980, reflecting the appearance of new products or services in the market. We use only those that are
} 
services from raw materials such as crude oil to end user consumables. We denote the 830 time-series data by $p_{\alpha}(t)$ where $\alpha=1,2, \cdots, 830(:=N)$ denotes the kind of goods and services, and $t=1,2, \cdots, 402$ stands for the month during the period from January, 1980 to June, 2013.

Before studying individual prices, let us take a look at the aggregate price indices. Fig. 1 (a) shows the time-series of monthly price indices from 1980 up to present (all indices; 2010 base). Note that the IPI are shown in a different scale (right axis) because it has much greater volatility than those of DCGPI and CPI. For reference, three epochs in which VAT was raised, in Aprils of 1989, 1997 and 2014 (VAT 3\%, $5 \%, 8 \%$ respectively) and the epoch of the Lehman shock in September 2008 are marked by vertical dashed lines in Fig. 1.

Fig. 1 (b) is the plot of annual (year-to-year) changes of the monthly aggregate price indices. The IPI has a different scale as depicted by the right axis for the reason explained above. One can observe that Japan suffered from deflation for a more than a decade from 1999 to 2013 in terms of domestic price indices, namely DCGPI and CPI.

We study the behavior of individual prices. We examine monthly changes of the individual price ${ }^{2}$ defined by

$$
r_{\alpha}(t):=\log _{10}\left[\frac{p_{\alpha}(t+1)}{p_{\alpha}(t)}\right] .
$$

Heterogeneity of micro prices found in the existing literature can be easily confirmed for the Japanese data we analyze. Table 1 shows the mean duration $d$ (in months) of the period during which individual prices remains unchanged for 39 groups of goods and services. The table also shows $\lambda$, the monthly frequency or probability that the price changes in a month (not directly observed). If one assumes that the prices can change at any instance of time with the constant probability, a simple Poisson process leads that $d$ is equal to $-1 / \ln (1-\lambda)$. Given $d$, the values for $\lambda$ in the table are estimated by this formula ${ }^{3}$.

listed every month during the whole period from January 1980 to June 2013 for consistency of our analysis.

${ }^{2}$ There are no seasonal adjustments in any of the individual prices we use. This is because only a limited number of them, such as clothing and vegetable, vary depending on seasons (Statistics Japan 2014). Applying seasonal adjustment on some selected individual prices while not doing so for others necessarily brings in some ad-hoc assumptions. They are not desirable for our analysis. Using the year-to-year rate of change is an alternative to seasonal adjustment and has an advantage of being free from ad-hoc assumptions. It, however, has a severe disadvantage of having a yearlong aftereffect from a big change, such as the introduction and raise of the consumption tax, and therefore are not adopted in our analysis.

${ }^{3}$ Assume that the price changes according to a homogeneous Poisson process with parameter $\theta$, namely a constant probability of change at any instance of time. For a realization of $n$ changes of the price at times $0 \equiv t_{0}<t_{1}<t_{2}<\cdots<t_{n} \equiv T$, the likelihood function is given by $L=\theta^{n} \exp (-\theta T)$, because the inter-occurrence times $T_{k}=t_{k}-t_{k-1}(k=1,2, \ldots, n)$ are independent and identically distributed by an exponential distribution with parameter $\theta$. The maximum likelihood estimate is then obtained by $\theta=n / T=1 / d$. On the other hand, the probability that the price changes in a month, $\lambda$, is related to the parameter $\theta$ by $\lambda=1-e^{-\theta}$ as easily shown. It therefore follows that $d=-1 / \ln (1-\lambda)$. See Basawa and Prakasa Rao (1980, Chap.6.2) for example. 


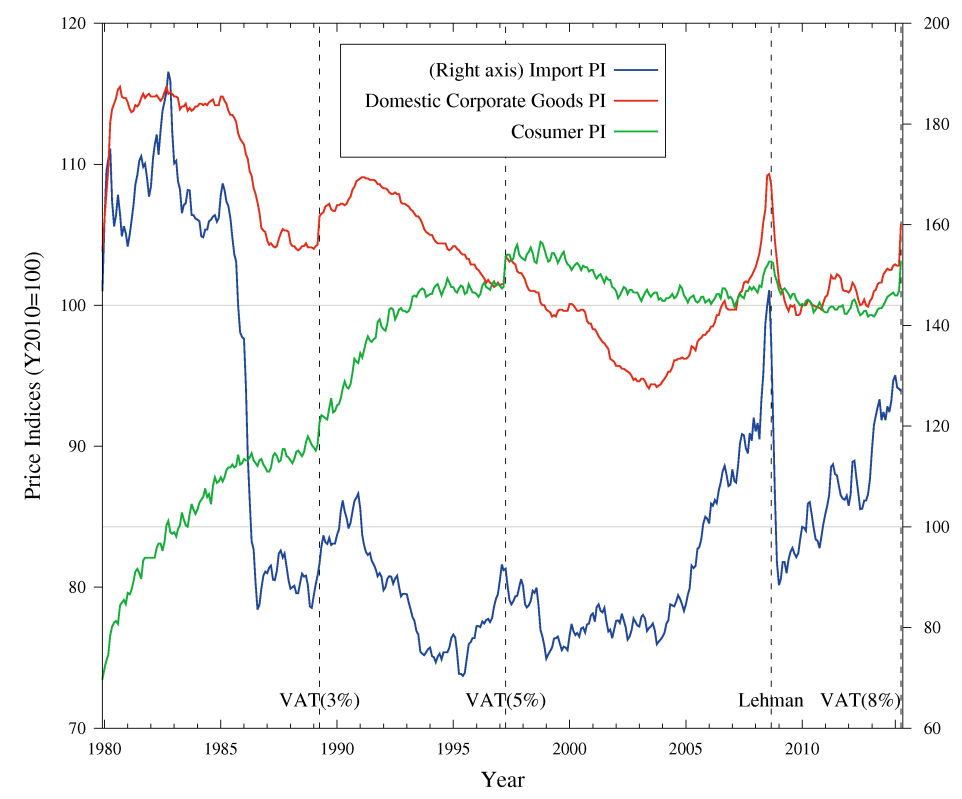

(a) Monthly price indices.

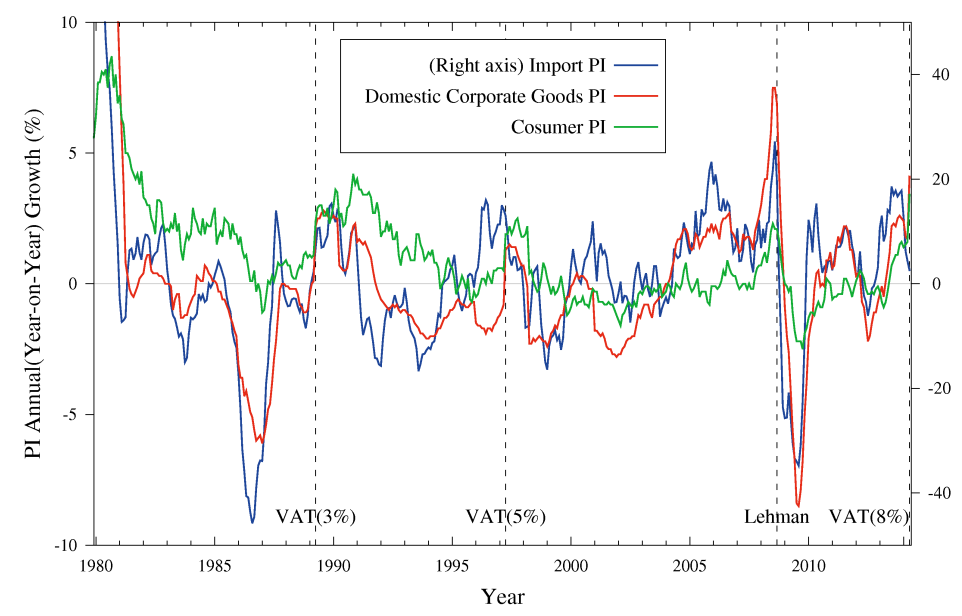

(b) Annual (year-on-year) changes in monthly price indices.

Figure 1. Time-series of monthly price indices (PI) for Import PI (blue and right-axis), Domestic Corporate Goods PI (red), and Consumer PI (green) from 1980 up to present (all indices; 2010 base). Dashed vertical lines correspond to the three months in which VAT was raised, namely April of 1989, 1997 and 2014 (VAT 3\%, 5\%, 8\% respectively), and September 2008 in which the Lehman Borthers went into bankruptcy.

The mean duration varies from 10 months for business machinery and transportation equipment to one month for food, cloths and most imported goods and materials. In between is 6 months for chemicals in DCGPI and services in CPI. On the whole, prices of imported goods and materials are very flexible. They are broadly consistent with the results obtained in previous works. 


\begin{tabular}{|c|c|c|c|c|}
\hline ID & CLASSIFICATION OF SECTOR & \#GOODS & MONTHS & FREQ \\
\hline \multicolumn{5}{|c|}{ IPI - Import PI } \\
\hline 01 & Foodstuffs \& feedstuffs & 17 & 1.04 & 61.80 \\
\hline 02 & Textiles & 6 & 1.26 & 55.25 \\
\hline 03 & Metals \& related products & 19 & 1.06 & 61.11 \\
\hline 04 & Wood, lumber \& related products & 3 & 1.02 & 62.66 \\
\hline 05 & Petroleum, coal \& natural gas & 8 & 1.04 & 61.94 \\
\hline 06 & Chemicals \& related products & 9 & 1.50 & 53.20 \\
\hline 07 & General purpose, production \& business oriented machinery & 2 & 1.14 & 58.47 \\
\hline 08 & Electric \& electronic products & 2 & 1.13 & 58.84 \\
\hline 09 & Other primary products \& manufactured goods & 9 & 1.09 & 60.15 \\
\hline- & ALL & 75 & 1.13 & 59.75 \\
\hline \multicolumn{5}{|c|}{ DCGPI - Domestic Corporate Good PI } \\
\hline 01 & Food, beverages, tobacco \& feedstuffs & 78 & 3.29 & 32.92 \\
\hline 02 & Textile products & 19 & 8.04 & 21.35 \\
\hline 03 & Lumber \& wood products & 8 & 3.16 & 33.45 \\
\hline 04 & Pulp, paper \& related products & 20 & 3.22 & 30.96 \\
\hline 05 & Chemicals \& related products & 55 & 6.32 & 24.65 \\
\hline 06 & Petroleum \& coal products & 11 & 2.01 & 42.88 \\
\hline 07 & Plastic products & 8 & 3.75 & 26.94 \\
\hline 08 & Ceramic, stone \& clay products & 25 & 5.12 & 24.62 \\
\hline 09 & Iron \& steel & 26 & 4.49 & 27.51 \\
\hline 10 & Nonferrous metals & 19 & 1.54 & 51.38 \\
\hline 11 & Metal products & 26 & 5.21 & 22.78 \\
\hline 12 & General purpose machinery & 20 & 6.13 & 19.24 \\
\hline 13 & Production machinery & 16 & 4.77 & 26.90 \\
\hline 14 & Business oriented machinery & 6 & 10.41 & 12.01 \\
\hline 15 & Electronic components \& devices & 5 & 2.22 & 37.59 \\
\hline 16 & Electrical machinery \& equipment & 20 & 4.65 & 22.79 \\
\hline 17 & Information \& communications equipment & 4 & 2.95 & 33.57 \\
\hline 18 & Transportation equipment & 11 & 10.26 & 10.78 \\
\hline 19 & Other manufacturing industry products & 15 & 8.87 & 16.91 \\
\hline 20 & Agriculture, forestry \& fishery products & 17 & 5.18 & 40.18 \\
\hline 21 & Minerals & 3 & 10.18 & 13.67 \\
\hline 22 & Electric power, gas \& water & 3 & 8.31 & 16.07 \\
\hline 23 & Scrap \& waste & 5 & 1.09 & 60.27 \\
\hline - & ALL & 420 & 4.95 & 28.37 \\
\hline \multicolumn{5}{|c|}{$\mathrm{CPI}$ - Consumer PI } \\
\hline 01 & Goods related to Food & 132 & 1.24 & 57.67 \\
\hline 02 & Goods of house materials, household utensils (incl. electronics) & 35 & 1.16 & 57.88 \\
\hline 03 & Goods of clothes \& footwear & 22 & 1.28 & 55.06 \\
\hline 04 & Goods of medical care & 11 & 1.55 & 48.09 \\
\hline 05 & Goods of automobiles, car equipments, misc. & 6 & 3.19 & 36.81 \\
\hline 06 & Goods related to education, culture, recreation \& misc. & 44 & 6.61 & 39.55 \\
\hline 07 & Services in CPI & 85 & 6.85 & 31.40 \\
\hline - & ALL & 335 & 3.41 & 47.79 \\
\hline
\end{tabular}

Table 1. List of IDs, classification of sectors, the numbers of goods, the durations and frequencies of price changes for the commodities of IPI, DCGPI and CPI. The sectors for IPI and DCGPI correspond to major groups based on the BOJ datasets. Those for CPI are classified by the authors partially based on the original classification and identities. Months is the mean duration between price changes, denoted by $d$. Freq is the constant monthly frequency of price changes or probability (in percent) that the price changes in a month, $\lambda$, estimated from $d$ based on a simple assumption of Poisson process, i.e., by $d=-1 / \ln (1-\lambda)$. 


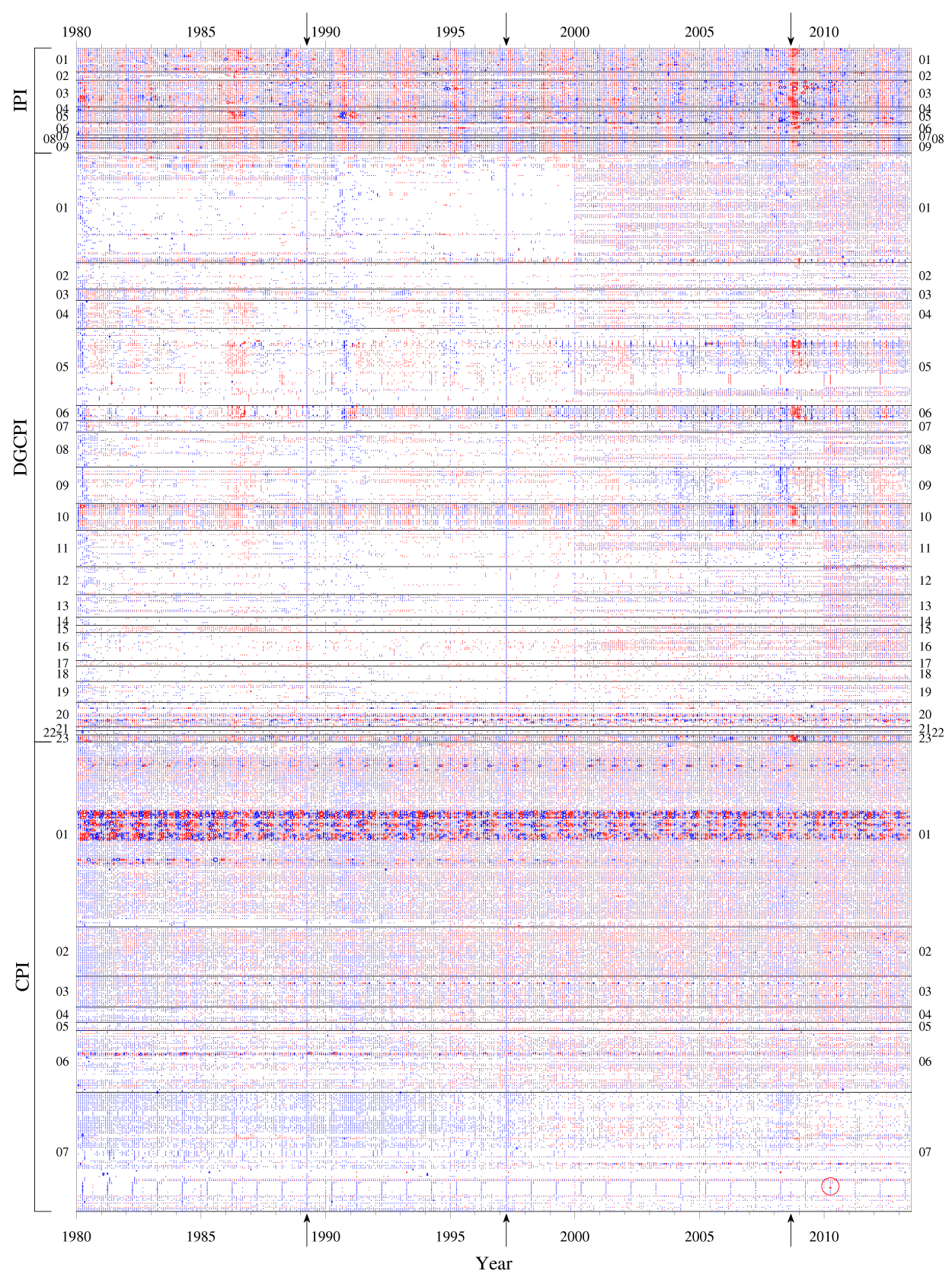

Figure 2. Monthly price changes $r_{\alpha}(t)$ for 75 individual goods comprising IPI, 420 goods for DCGPI, 335 goods for CPI from January 1980 to June 2013. Segments denoted by labels starting from "01" in each PI are classification of sectors. Blue and red colors correspond to positive and negative changes (ups and downs), respectively. Blank areas correspond to no monthly change, $r_{\alpha}(t)=0$. Each circle has a radius proportional to the absolute magnitude of change. Three arrows are drawn at the epochs of VAT 3\% (Apr 1989), VAT 5\% (Apr 1997), Lehman shock (Sep. 2008). 


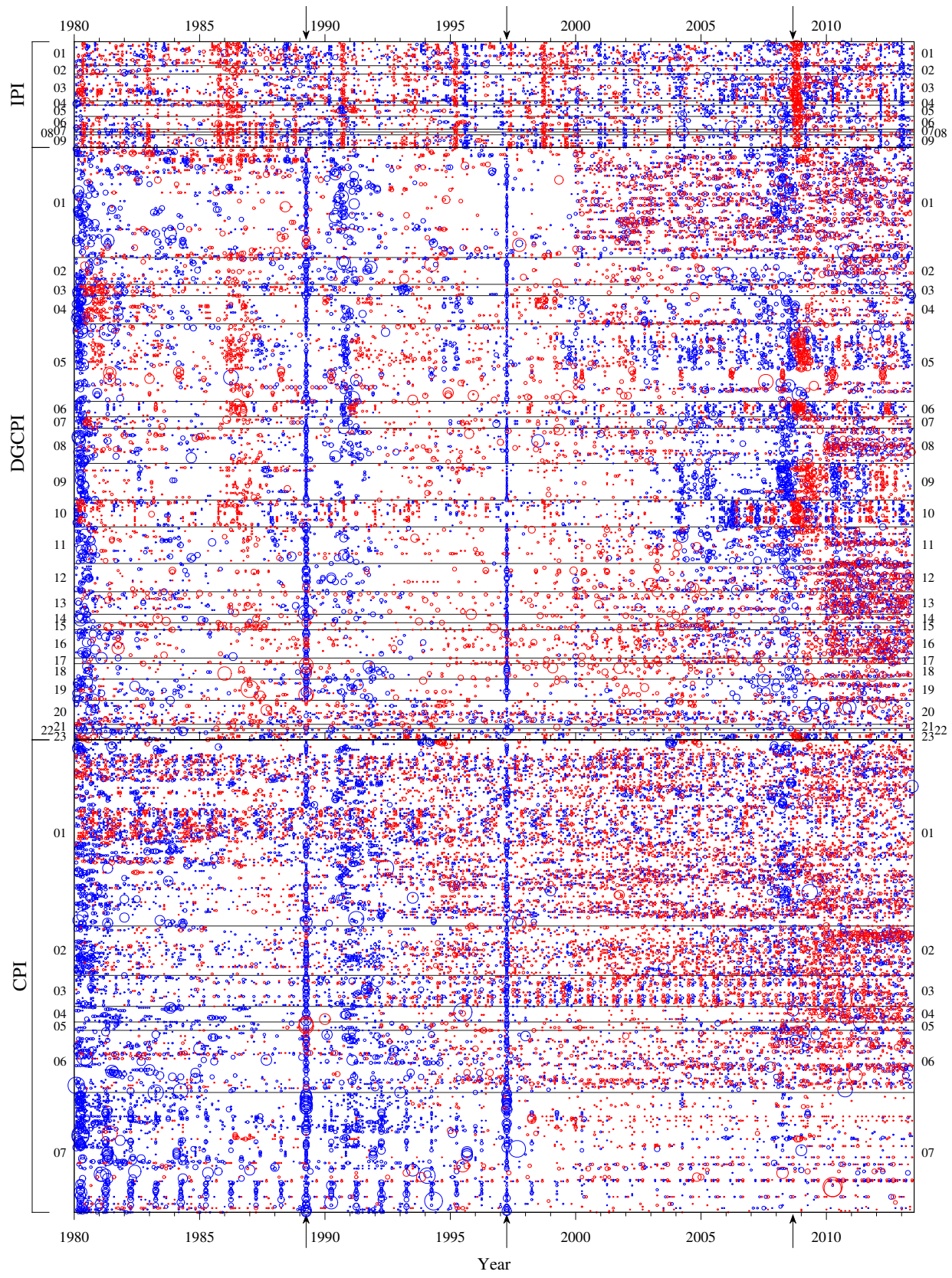

Figure 3. Normalized price changes $w_{\alpha}(t)$ calculated from the monthly changes $r_{\alpha}(t)$ in Fig. 2 Blue and red colors correspond to $w_{\alpha}(t)>w_{*}$ and $w_{\alpha}(t)<-w_{*}$, respectively, where the threshold is set as $w_{*}=1.0$. Blank areas correspond to no "significant" change in the sense that $\left|w_{\alpha}(t)\right|<w_{*}=1.0$. The segments such as "01" in each PI, the colors/radius, and the arrows have the same meaning given in Fig. 2 
Micro prices of individual goods and services have different volatilities. They must reflect differences in industrial organization and the nature of goods and services. Prices of imported oil and other materials are globally determined in wellorganized auction markets. Import prices are also affected by changes of the exchange rate. To take into account these differences in volatility, in what follows, we consider the normalized price change. Denoting by $\left\langle r_{\alpha}\right\rangle_{t}$ and $\sigma_{\alpha}$ the sample average and standard deviation of the time-series $r_{\alpha}(t)$, respectively, we define the normalized time-series by

$$
w_{\alpha}(t):=\frac{r_{\alpha}(t)-\left\langle r_{\alpha}\right\rangle_{t}}{\sigma_{\alpha}}
$$

All 830 series of $w_{\alpha}(t)$ are found to be stationary by the Dicky-Fuller test (Dickey and Fuller, 1979) and the Phillips-Perron test (Phillips and Perron, 1988) by the use of Mathematica.

Seeing is believing. Fig. 2 shows monthly changes of 830 individual prices of the goods and services, $r_{\alpha}(t)$, for the period from January 1980 to June 2013. Blue and red colors of each point indicate positive and negative changes, $r_{\alpha}(t)>0$ and $r_{\alpha}(t)<0$, respectively. Blank space means that the price did not change, namely $r_{\alpha}(t)=0$. The portions of "IPI", "DCGPI" and "CPI" indicated on the vertical axis in Fig 2 correspond, respectively, to 75 goods comprising IPI, 420 goods for DCGPI, and 335 goods and services for CPI. These individual points are grouped into the sectors that they belong to. The lists of sectors for IPI, DCGPI, CPI are summarized in Table 1 .

W examine "spatio-temporal patterns" of the individual price changes. Fig. 3 shows the normalized changes $w_{\alpha}(t)$ defined by Eq.(2). Fig. 3 focuses on "significant" changes of prices in the sense that the data for $\left|w_{\alpha}(t)\right|<1$, namely changes smaller than one standard deviation, are shown as blank space. Blue and red colors of each point indicate significant positive and negative changes, namely $w_{\alpha}(t)>1$ and $w_{\alpha}(t)<-1$, respectively.

Fig. 3 demonstrates that the simultaneous changes of individual prices or the synchronization occasionally occur without any clear periodicity. The April 1989 and the April 1997 are two examples of extreme synchronization as indicated by the arrows in Fig. 3. In Japan, the three percent value added tax (VAT) called the consumption tax was introduced in April 1989, and the tax rate was raised from three to five percent in April 1997. Almost all the prices were raised then. Note, however, that individual prices were not mechanically raised by three and two percent, respectively. Evidently, many firms found good opportunities to adjust their prices when the VAT rate was changed. We plot the rate of changes of the individual prices for the two periods around April, 1989 and 1997 in Figs.45.

At the time of the introduction of the consumption tax, we observe that most CGPI were raised by $3 \%$, while CPI shows wide distribution of price changes around the $3 \%$ mark. In fact, the average rate of change in CPI are $2.47 \%$ and $1.68 \%$, respectively, for the two periods in question. The reason would be that a majority of suppliers of consumer goods were afraid of loosing sales and did "absorb" the consumption tax raise. On the other hand, DCGPI are for intermediate goods 
(a)

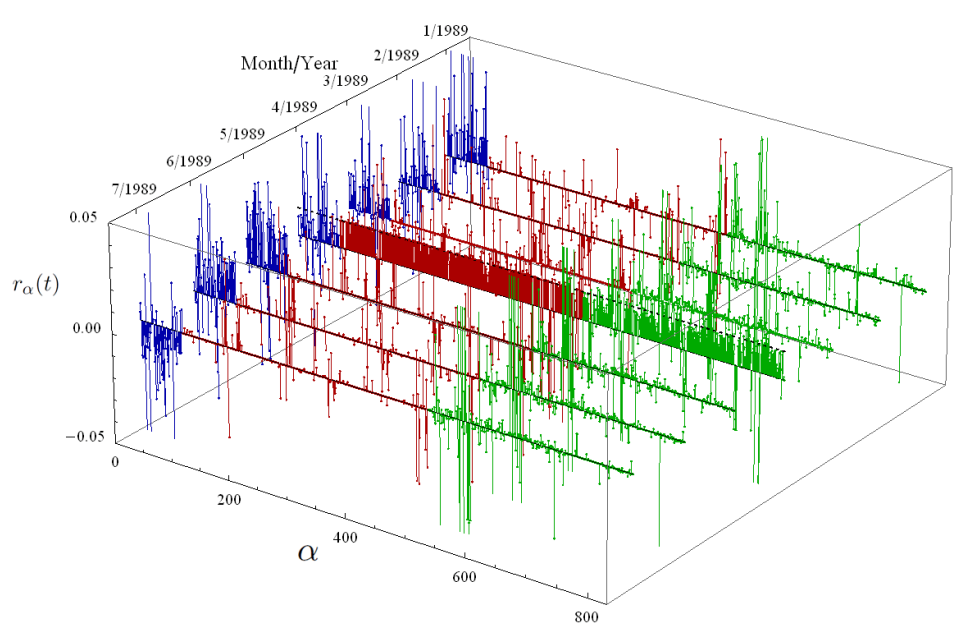

(b)

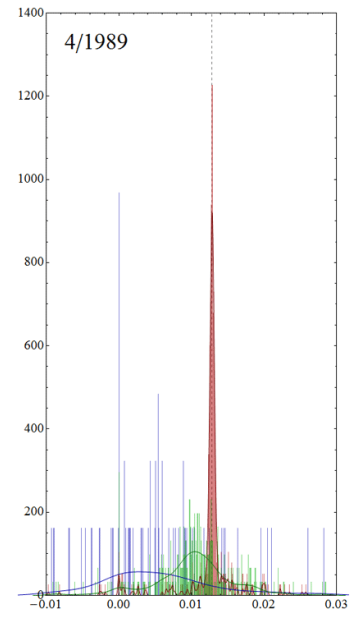

Figure 4. Behavior of the rate of change $r_{\alpha}(t)$ at the time of introduction of the consumption tax. The plot (a) shows the rate of change for the seven months surrounding April 1989, when the consumption tax of $3 \%$ was introduced. IPI is shown in blue, DCGPI red, and CPI in green. The dashed horizontal line on $4 / 1989$ corresponds to the $3 \%$ raise $\left(\log _{1} 0(1.03)=0.0128\right)$. Plot $(\mathrm{b})$ shows the histogram of the rate of change on April 1989 for IPI, CGPI, CPI in the same color scheme as in (a), and the smoothed histogram in the same manner. The dashed vertical line corresponds to $3 \%$ raise.

traded between firms, and there was no problem for adding consumption tax onto the existing prices $4^{4}$

One can quantify the degree of synchronization of micro price changes by examining the numbers of positive, negative and zero price changes for each month. Let us denote such numbers by $n_{+}(t), n_{-}(t), n_{0}(t)$, and the sum of them is the total number of goods and services, $N$. Fig. 6 (a), (b), (c) show the fractions $n_{+}(t) / N$, $n_{-}(t) / N, n_{0}(t) / N$, for IPI, DCGPI and CPI (from top to bottom), respectively. Not to mention volatile IPI, one can observe that DCGPI and CPI prices are also raised or lowered in time-varying way. The number of prices that are raised is larger than those that are lowered under (even mild) inflation, while the converse is true under deflation. For example, in the plot for CPI, the fraction $n_{-}(t) / N$ exceeds $n_{+}(t) / N$ persistently from 1999 up to 2007 when deflation continued.

In what follows, we examine two periods in details: (a) the post-Plaza Agreement yen appreciation, 1985-88, (b) the pre- and post-Lehman Brothers bankruptcy, 200709 .

\footnotetext{
${ }^{4}$ At the time of raise to $5 \%$ from $3 \%$, we observe that most of CGPI were raised by $1.94 \%$, which comes from $1.05 / 1.03=1.0194$. It should be noted this is not $2 \%$ as seen in Fig 7 because if we denote the price index at the time of $3 \%$ sale tax by $r_{3}$, the pretax price of the good/service is $r_{3} / 1.03$ and by adding the $5 \%$ consumption tax, it will be $r_{3} / 1.03 \times 1.05$. on the other hand, CPI shows distribution peaked at around $1.94 \%$ and skewed to larger values. This differs from 4/1989.
} 
(a)

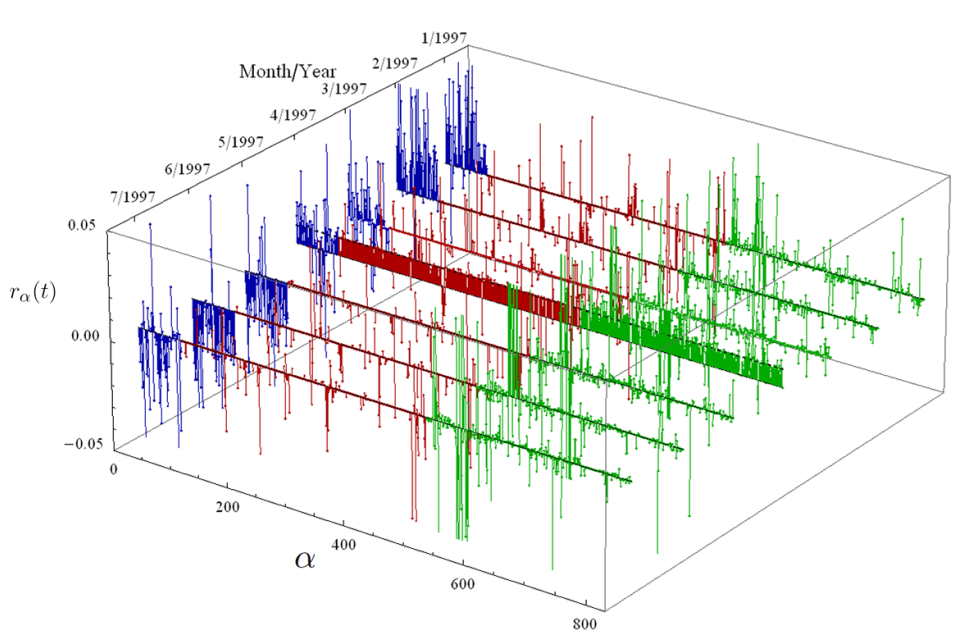

(b)

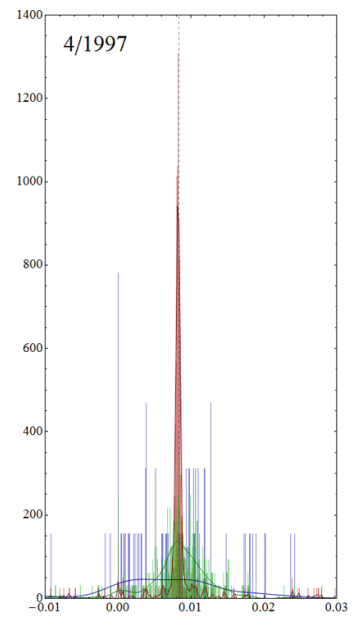

Figure 5. Behavior of the rate of change $r_{\alpha}(t)$ at the time of the raise of the consumption tax from $3 \%$ to $5 \%$. The plot (a) shows the rate of change for the seven months surrounding April 1997, when the consumption tax of raised to 5\%. The dashed horizontal line on 4/1997 shows the $1.94 \%$ raise. Plot (b) shows the histogram of the rate of change on April 1997, with the dashed vertical line corresponding to $1.94 \%$ raise. 
(a) IPI

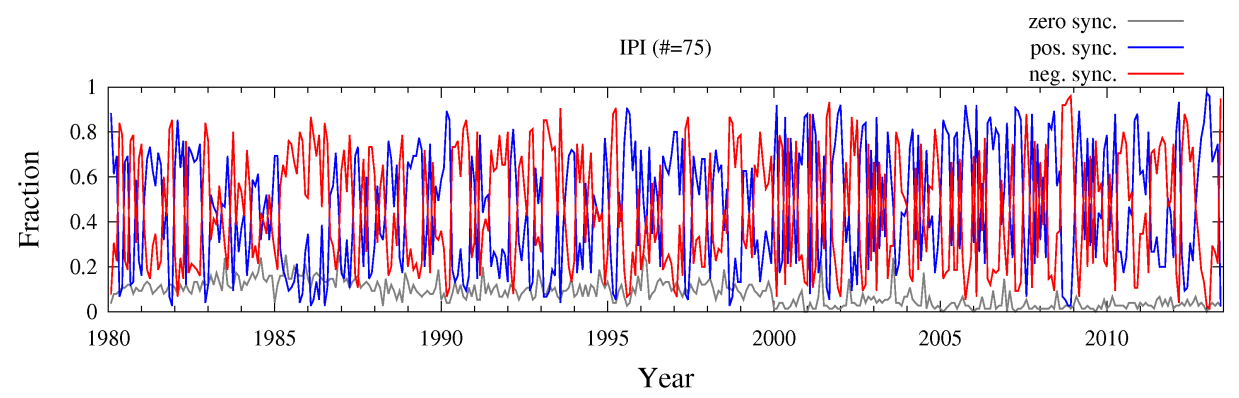

(b) DCGPI

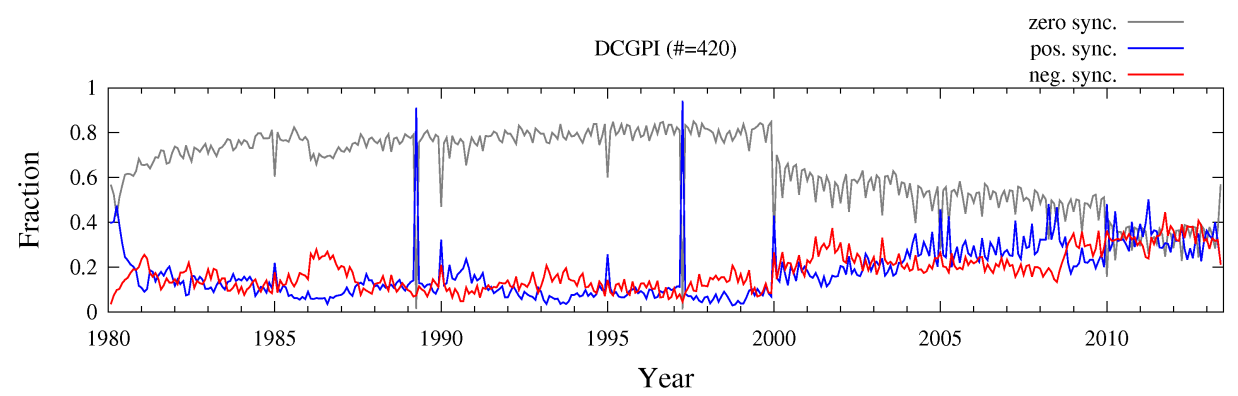

(c) CPI

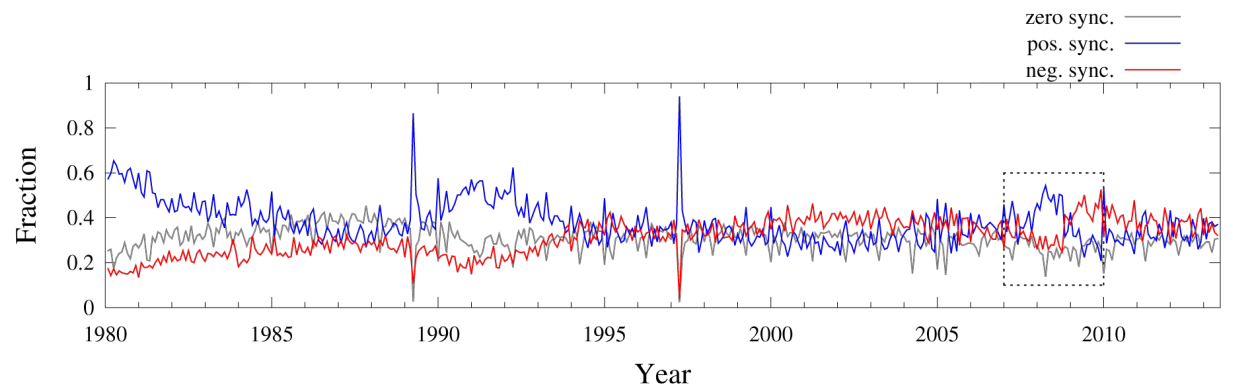

Figure 6. The fraction of the numbers of goods and services for which we observe positive (blue), negative (red) and zero (gray) price changes, respectively. From top to bottom: (a) IPI, (b) DCGPI, (c) CPI. 


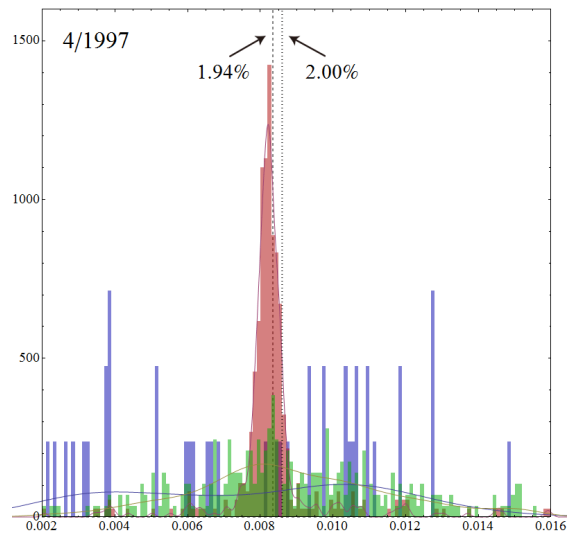

Figure 7. Detailed view of the histogram of the rate of change $r_{\alpha}(t)$ on April 1997, with the dashed vertical line showing $1.94 \%$ and the dotted vertical line show $2.00 \%$. 


\section{(a) The Post-Plaza Agreement Appreciation of the Yen, 1985-1988}

When the Plaza Agreement was signed on September 22nd, 1985, the exchange rate was 240 yen per dollar. After the Agreement, the yen appreciated sharply, and by the end of 1987, had reached 120 yen per dollar. The sharp appreciation of the yen within two years significantly affected prices.

Fig. 8 is the enlargement of Fig. 3 for the period from September 1985 to August 1988, with the threshold=2.0. Blue and red circles correspond to ups and downs, respectively, with a radius showing the magnitude of a change. Following the Plaza Agreement in September 1985, most import prices kept declining until September, 1986. Prices of many intermediate goods in DCGPI also declined (See Table 1 for the identification of products). For the next one year (September 1986 - September 1987), import prices stopped falling, whereas prices of intermediate products in DCGPI continued to fall. In contrast, for the whole period, most consumer prices rose rather than declined with an exception of foodstuffs most of which use imported goods.

Evidently, the impulse to prices during this period was the sharp appreciation of the yen from 240 per dollar to 120 . Import prices fell in the first year following the Plaza Agreement in September, 1985, and with one year lag, prices of intermediate good in DCGPI started falling. CPI kept rising albeit only mildly. We note that during Sept. 1985 - May, 1987, the growth rate of money had been very stable at the rate of 8-9 percent. Plainly, money cannot explain the changes of prices during this period. 


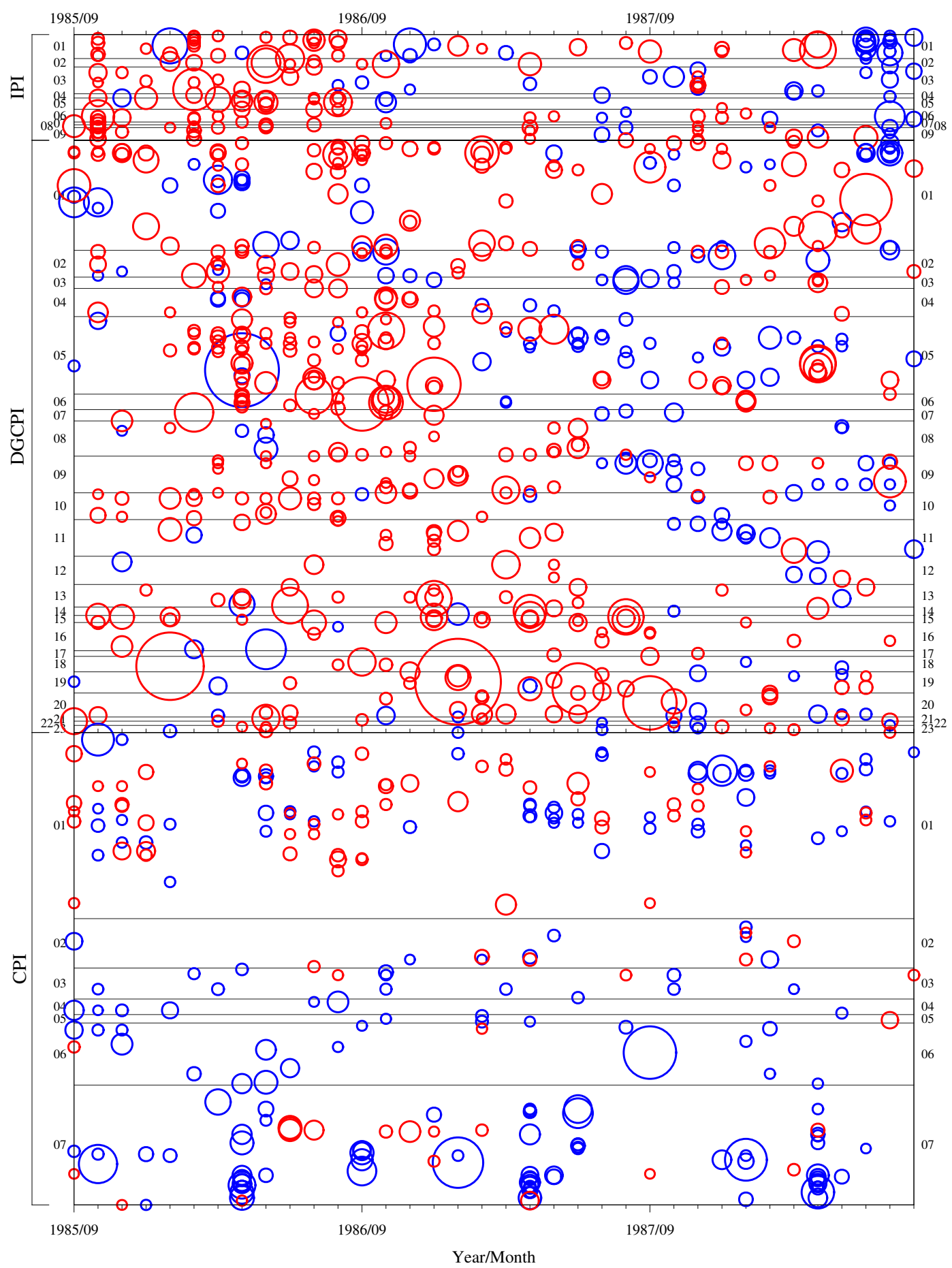

Figure 8. Enlarged view of Fig. 3 for the period of the post-Plaza accord from September 1985 to August 1988, when the yen appreciated sharply. Normalized price changes $w_{\alpha}(t)$ are shown with blue and red colors for $\left|w_{\alpha}(t)\right| \geq w_{*}=2.0$. Each circle has a radius proportional to the absolute magnitude of change. Blank areas correspond to $\left|w_{\alpha}(t)\right|<w_{*}$. 


\section{(b) The Great Recession, 2008-2009}

The year 2008, shown in Fig. 9, provides us with an interesting case. In the first half of the year, import prices and prices of intermediate products in DCGPI significantly went up. In CPI, food prices also rose. Deflation appeared to change into mild inflation during this period.

The bankruptcy of the Lehman Brothers in September 2008 turned the tide. The fraction of price decline suddenly went up (Fig. 9). This sudden change is clearly seen in Figure 10 (a) and (b) which enlarges Fig. 3 for the period during 2007-09. The figures show how mild inflation up to the first half of the 2008 suddenly changed to deflation in the course of the Great Recession triggered by the bankruptcy of the Lehman Brothers in September, 2008. Evidently, changes in price during this period have little to do with money, because the growth rate of money during May 2008 March 2009 had hardly changed within narrow limits of 1.9 and 2.4 percent. They basically reflect a fall of real economic activity, namely the Great Recession.

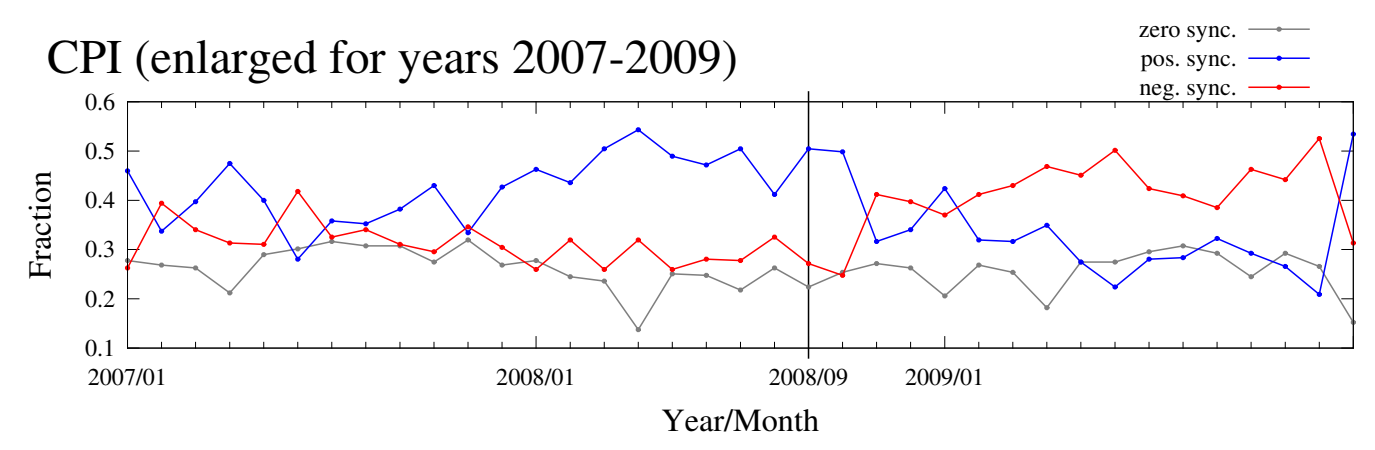

Figure 9. The fraction of the numbers of goods and services for which we observe positive (blue), negative (red) and zero (gray) price changes, respectively. for the three years from 2007 to 2010. 
(a)

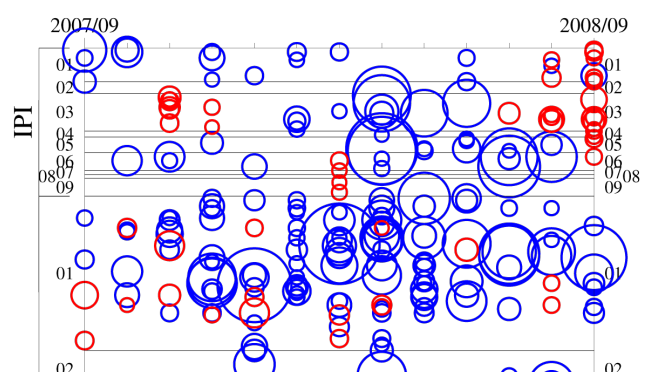

0282

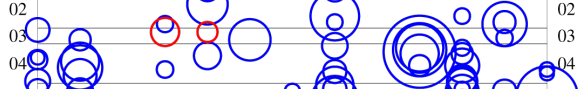

Q8 0 o@

${ }_{05} 80$ o 8 (2)

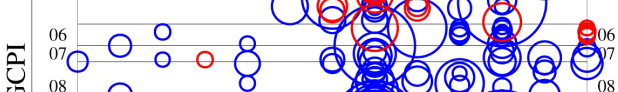

- 80000020

${ }^{10} \mathrm{O} \circ \mathrm{O} 0 \mathrm{~b}(\mathrm{~s})$

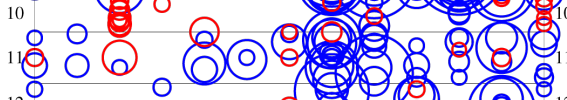

${ }_{12}{ }_{12} 8 \bigcirc 8$ (8) ${ }_{013}^{8}$

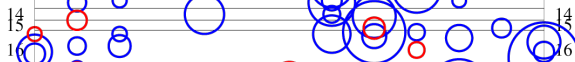

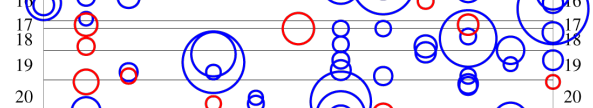

${ }_{22210}^{20} 0$ (3) (2) 00

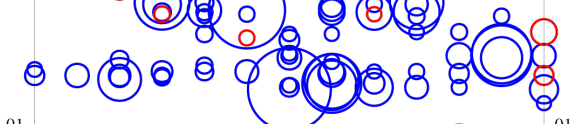

${ }^{01} 8$ \% $8 \circ \bigcirc \circ 8^{01}$

- \& 8 \% 8 \%

$\circ 88.808$ a 888

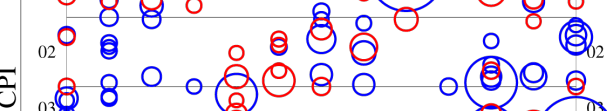

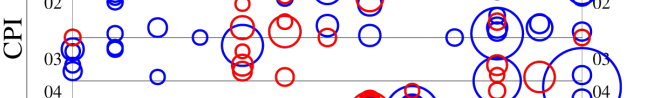

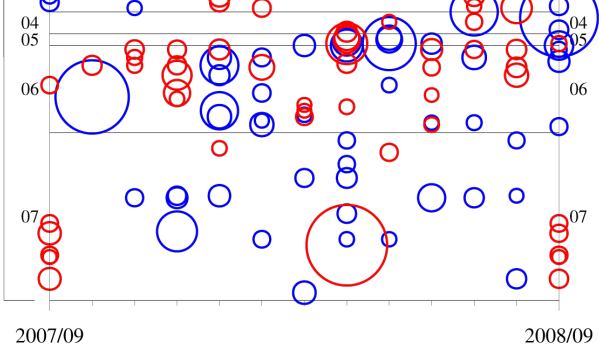

(b)

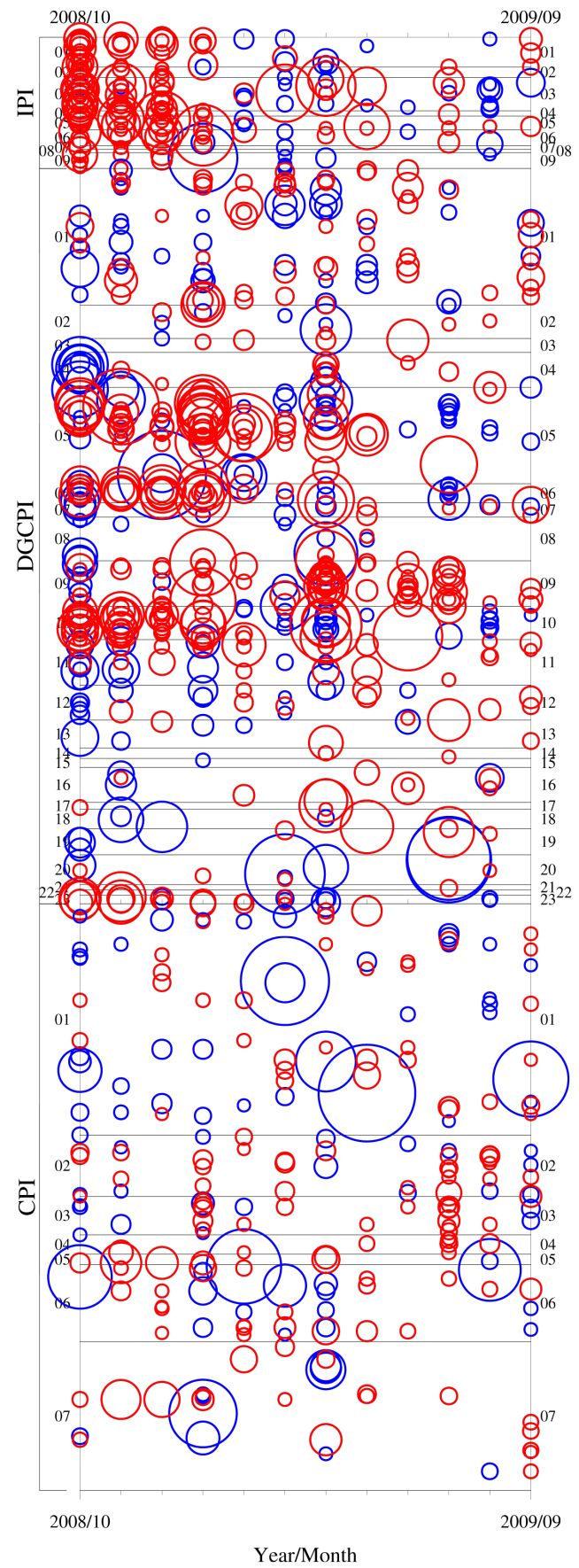

Figure 10. Enlarged view of Fig. 3 for the periods (a) one year before the Lehman shock in September 2008 and (b) one year after the shock respectively. The threshold $w_{*}$, colors and radius of circles are the same as given in the caption of Fig. 8 


\section{Summary}

The analyses in this section demonstrate the following important points.

1. The average frequency of price change of individual goods or service provides only a very limited information on the behavior of aggregate price because price change is not time homogeneous. The year 2008 is a good example. In the first half of the year, many prices were raised, but the bankruptcy of the Lehman Brothers turned the tide, and afterwords, many prices declined, some significantly. In other times, most prices simply remained unchanged for a long time period.

This fact rejects the popular assumption that the firm's price changes strategy is time-invariant. For example, Calvo (1983) assumes that for each firm, an opportunity to change its price arrives at random with a given probability, while others in the more recent literature assume that the hazard rate of price is time-invariant. More generally, the existing literature focuses on cross-sectional distribution of micro prices, and assumes that it is given and time-invariant. We note that micro optimization exercise results in a particular pattern of price setting which is time-invariant. This assumption of time-invariance of price setting is not borne out by data. Instead, it is important to explore what macro variables drive individual prices to synchronized actions.

2. In order to fully understand the behavior of aggregate price, we must explicitly consider subsets or clusters of prices, not just a single macro-group of prices. For example, look at Fig 3 for the period during 1995-2000 vertically. Except for April,1997 when VAT was raised, prices of some goods went up or down in clusters while others remained unchanged.

This point casts doubt on the existing theories of price setting such as menu cost and contract models. In most theoretical models, an individual firm is assumed to strategically set or reset its price considering the behaviors of all the other firms. It is commonly assumed that firm $j$ is interested in $P_{j} / P$ where $P_{j}$ is the firm $j$ 's price and $P$ is the aggregate price index. In other words, it is routinely assumed that the universe in which each firm optimizes is the economy as a whole. However, the behavior of individual prices shown in Fig. 3 does not support this presumption; it shows that there is a significant tendency that a cluster of prices change together while at the same time prices which belong to other clusters do not. The standard theoretical model takes the macroeconomy as if it were a single industry or a group of retailers in a region. Such a model may serve for the purpose of industrial organization, but does not fit the purpose of macroeconomics and monetary policy.

Generally, we can consider how $N$ commodities' prices are determined by $J$ firms. A firm changes the prices of goods and services which it produces in response to the changes in other prices. However, firm is not interested in all prices, but only in a subset of prices. Obvious examples are prices of intermediate goods and services used in production, and also prices of close substitutes produced by rival firms. The 
response is not usually taken based on the single information of aggregate price $P$ in a synchronized way, but on a partial information among the $N$ prices that are relevant to the firm. Gordon (2011, p.32-33) points out the same problem as follows.

"(Recent research on inflationary expectations is) flawed because it placed the information barriers in the wrong place, in an inability to perceive costless macro information, instead of where the information barriers really exist, at the micro level of costs and supplier-producer relationships. Producers of final goods are unable to perceive cost increases of crude and intermediate materials that may be in the pipeline, and they have no choice but to wait until they receive notification of actual cost changes (with the exception of crude materials like oil where prices are determined in public auction markets). ‥ A fundamental source of persistence is not just explicit wage contracts as analyzed by Taylor, but also explicit or implicit price contracts between suppliers and producers of final goods. Even without contracts, persistence and inertia are introduced by lags between price changes of crude materials, intermediate goods and final goods. For some goods, e.g. cars or aircraft, there are literally thousands of separate intermediate goods, and most of these are made up of further layers of intermediate goods."

Setting the price is, of course, a very important economic decision by firm. To do so, the firm must first define a subset of prices which together with its own price, crucially affect its sales, production costs, and profitability. The obvious candidates are prices made by the rival firms producing the same product or close substitutes, and also prices of materials. The important point is that this subset of prices relevant for the firm's price setting does not encompass all the prices in the economy as a whole. On the other hand, such subsets of prices overlap each other. Thus arises nontrivial price dynamics which takes time. Fig. 11 illustrates this problem. The standard assumption in macroeconomics is that firms take the macroeconomy as common universe, and that they optimize in it. This assumption (Fig. 11(a)) is not borne out by data. Firms belong to their respective small universes. Such micro universes overlap with each other (Fig. 11(b)). This structure produces dynamics by itself. In Section IV], we will analyze such dynamics by a new analytical method.

It is too simple to infer that without menu cost or nominal contract, prices can swiftly change. Most macroeconomists may take the following statement for granted.

"Consider a monetary shock. The efficient response to a doubling of the money supply is for all prices to double immediately and all real quantities to remain unchanged." (Nakamura and Steinsson, 2013).

Most business people would be appalled at such statement, however. Most likely, the micro information set on which firm sets its prices would not contain money supply. Rather, prices affect prices with leads and lags. The existing literature almost completely misses this important lead/lag relationships among micro prices. The analysis in Section IIIdemonstrates the importance of such interactions of micro 
(a)

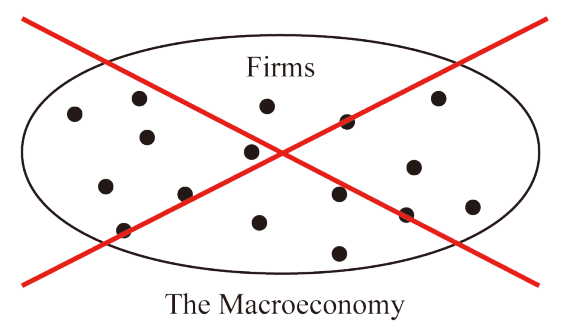

(b)

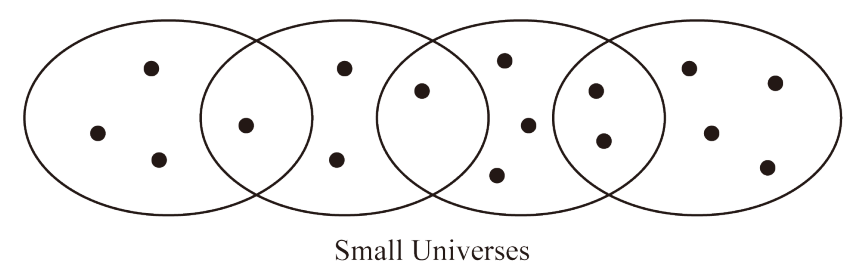

Figure 11. Firms determine the prices of their own goods and services in response to the changes of prices of other goods and services. Firm is not interested in all the prices, but only in a subset of prices. Such subsets intersect each other. This structure produces dynamics by itself.

prices. To better understand the behavior of the aggregate price, we must uncover such dynamics by taking advantage of micro price data. 


\section{Autocorrelations of Prices}

In macroeconomics and monetary policy, we are interested mainly in changes of the aggregate price index such as CPI. In this section, we study autocorrelations of the rate of change of price, specifically autocorrelations of the rate of change of price relative to the twelve months earlier. That is, we examine $\pi_{\alpha}(t)$, the rate of change of price $p_{\alpha}$ at time $t$ given by

$$
\pi_{\alpha}(t)=\log p_{\alpha}(t)-\log p_{\alpha}(t-12) .
$$

Stationarity of the year-to-year change of CPI, $\pi_{\alpha}(t)$ was confirmed by conducting the Dickey-Fuller F test with no drift term; the $p$-value takes 0.00102 much smaller than the standard significance level $\alpha=0.05$. Also the year-to-year change of 320 prices out of the totally 335 CPI-constituting prices passed the unit-root test.

Figure 12 shows the autocorrelations of individual prices of 335 goods and services which comprise CPI. The autocorrelations of micro prices considerably differ across goods and services. However, they share a clearly observed common pattern. Namely, the autocorrelations almost linearly decline up to 12 months, and then flatten afterwords.

We note that this pattern is to be expected if monthly $\log p_{\alpha}(t)$ follows random walk:

$$
\log p_{\alpha}(t)-\log p_{\alpha}(t-1)=\epsilon_{t}
$$

where $\epsilon_{t}$ is white noise. In this case, we observe

$$
\pi_{\alpha}(t)=\log p_{\alpha}(t)-\log p_{\alpha}(t-12)=\epsilon_{t}+\epsilon_{t-1}+\cdots+\epsilon_{t-11} .
$$

The autocorrelation function $\phi_{\alpha}(t)$ for $\pi_{\alpha}(t)$ in the random walk model is therefore calculated as

$$
\phi_{\alpha}(t)=1-\frac{t}{12}(0 \leq t \leq 12) \text {, }
$$

with $\phi_{\alpha}(t)=0$ beyond $t=12$. This correlation stems from accumulation of random shocks. Figure 12 suggests that monthly individual prices follow random walk, and that micro shocks to individual prices are permanent.

The heavy tail observed in Fig. 12 originates from the 15 exceptional prices which fail the unit root test for stationarity. Particularly important is the imputed rent, whose share in CPI is 38 percent, by far the largest. We will show that our results for the collection of micro prices stand out if imputed rent is excluded.

Next, we examine the autocorrelation of CPI. Figure 13 compares (a) the autocorrelation function $\phi(t)$ of CPI with (b) the weighted average $\phi_{\text {self }}(t)$ of autocorrelations of 335 micro prices defined by

$$
\phi_{\text {self }}(t)=\sum_{\alpha} w_{\alpha} \phi_{\alpha}(t) .
$$

Here, the weight $w_{\alpha}$ is given by

$$
w_{\alpha}=\frac{g_{\alpha}^{2} \sigma_{\alpha}^{2}}{\sum_{\alpha} g_{\alpha}^{2} \sigma_{\alpha}^{2}}
$$




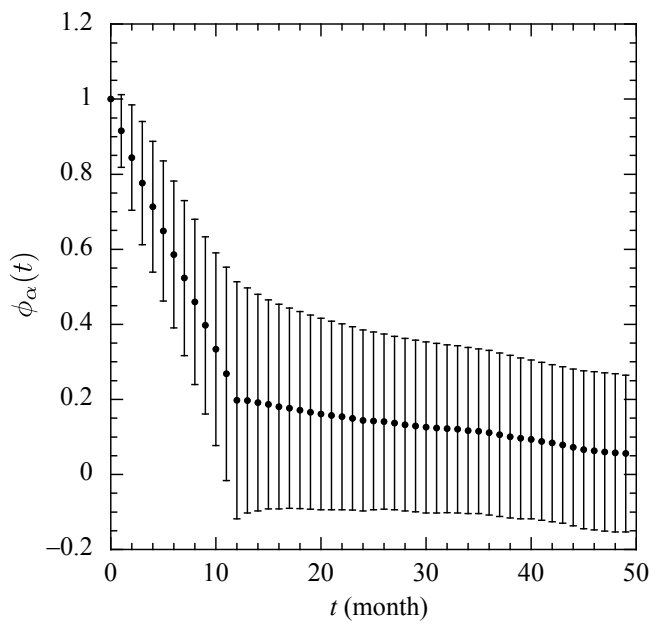

Figure 12. Autocorrelation functions $\phi_{\alpha}(t)$ for individual prices of 335 goods and services comprising CPI. The mean (dot) of $335 \phi_{\alpha}(t)$ 's with the standard deviation of their distribution (error bar) is plotted at every time difference.

with its variance $\sigma_{\alpha}^{2}$ and statistical weight $g_{\alpha}$ in CPI.

The autocorrelation of the aggregate price index, CPI has a very different pattern from those of micro prices; it follows an exponential decay. It means that the aggregate price index contains substantial long memory:

$$
\phi(t)=\exp (-t / \tau)
$$

In contrast, as explained by Eq. (6), the autocorrelations of individual prices have comparatively short memory.

The relation between autocorrelations of CPI and micro prices is formally as follows 5

$$
\left\langle\pi\left(t_{0}\right) \pi\left(t_{0}+t\right)\right\rangle_{t_{0}}=\sum_{\alpha} g_{\alpha}^{2}\left\langle\pi_{\alpha}\left(t_{0}\right) \pi_{\alpha}\left(t_{0}+t\right)\right\rangle_{t_{0}}+\sum_{\alpha \neq \beta} g_{\alpha} g_{\beta}\left\langle\pi_{\alpha}\left(t_{0}\right) \pi_{\beta}\left(t_{0}+t\right)\right\rangle_{t_{0}}
$$

where

$$
\pi(t)=\sum_{\alpha=1}^{n} g_{\alpha} \pi_{\alpha}(t) .
$$

Figure 13 a corresponds to the left-hand side of Eq. 10 whereas Fig. 13b, to the first term on the right-hand side of Eq. (10). In other words, the weighted average of autocorrelations of micro prices shown in Fig. $13 \mathrm{~b}$ excludes the effects arising from interactions of micro prices with leads and lags, the second term on the right-hand

\footnotetext{
${ }^{5}$ The aggregate price index defined by Eq. (11) and the official CPI are different because the former uses a subset of goods and services in CPI; specifically, we use only 335 long-lived prices selected out of total 593 prices comprising the official CPI (as of June 2014). However, in Fig 13 . we see that the resulting autocorrelation function of the aggregate price index is virtually identical to that of CPI over initial 20 months.
} 
side of Eq. 10). To the extent that Figs. $13 \mathrm{a}$ and $13 \mathrm{~b}$ are significantly different, we must take into account interactions of micro prices with leads and lags for our fully understanding behavior of aggregate price index.
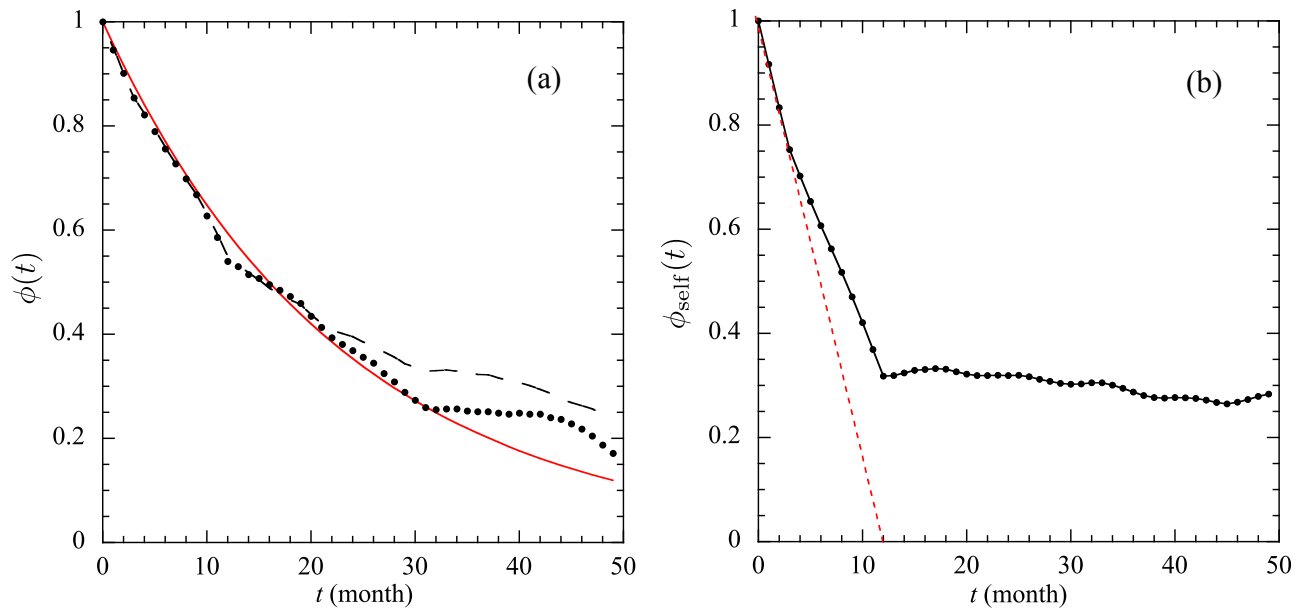

Figure 13. Autocorrelation function for CPI with all items (a), compared with weighted average of the autocorrelation functions for the individual prices (b). The solid curve in panel (a) shows an exponential decay form fitted to the numerical results (dots) for $t \leq 24$; its characteristic decay time $\tau$ is 23.1 months. The dashed curve in panel (a) depicts the autocorrelation of the aggregate price index obtained through Eq. 111). The dotted line in panel (b) shows Eq. (6).

We examine the statistical significance of interdependence of individual prices. For this purpose, we prepare a null model by randomly rotating time-series of individual prices in the time direction with a periodic boundary condition imposed. This randomization procedure destroys only cross-correlations involved in the original data, leaving autocorrelations as they are. That is, it is mathematically equivalent to omitting the second term on the right-hand side of Eq. 10 for time-series data (theoretically data of infinite length). Details of the data shuffling method, referred to as rotational random shuffling (RRS), are given later. By repeating the RRS, we generated 100,000 samples to evaluate statistical variations of the autocorrelations of the weighted average of individual prices; the statistical fluctuations arise from finiteness of the time-series data. In Fig. 14, their median, lower $5 \%$ level, and upper $5 \%$ level are compared with the autocorrelation function of CPI. We can first confirm that the median agrees well with $\phi_{\text {self }}(t)$ in Fig. $13 \mathrm{~b}$ as it is expected. We then compare autocorrelations of CPI to the upper $5 \%$ level in the null model. They lie out of the $5 \%$ level for $t \lesssim 12$. We thus conclude that interdependency among individual prices is statistically significant at the $95 \%$ level of confidence.

We carried out the same test for autocorrelations of CPI and individual prices excluding imputed rent. The imputed rent whose share in CPI is $38 \%$ fails to pass the unit root test. By construction, the imputed rest remains unchanged for several months. The results are shown in Figs. 15 and 16 . They strengthen our case.

In summary, the analyses of autocorrelations demonstrate that interdependence of micro prices with leads and lags plays an important role in determining the rate of 


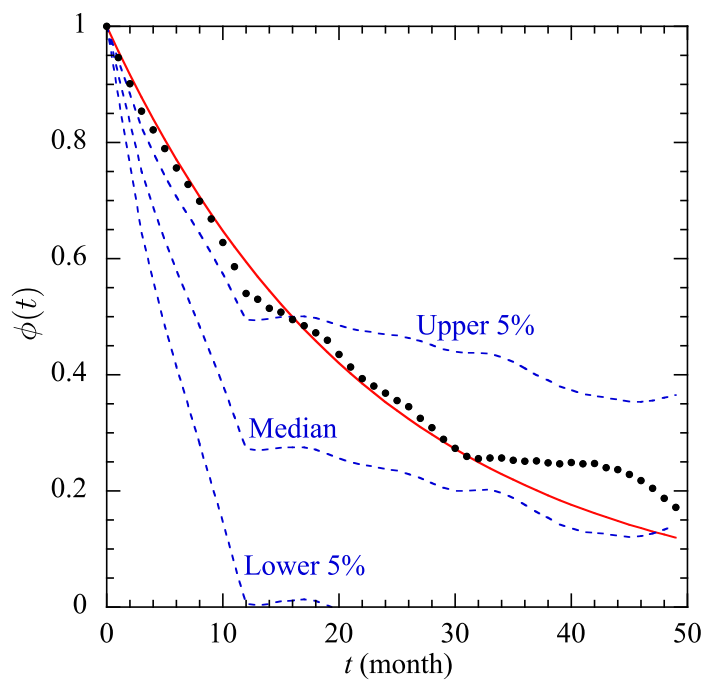

Figure 14. Test of statistical significance for interdependency of individual prices. The autocorrelation of CPI with all items as shown in Fig. $13 \mathrm{a}$ is compared with statistical variations of the weighted average of the autocorrelations for individual prices which are randomly rotated in the time direction (median, lower $5 \%$ level, and upper $5 \%$ level are shown by dotted curves); the number of samples is 100,000 . This shuffling provides us with a null hypothesis by destroying cross-correlations among prices with their autocorrelations preserved. The degree of the autocorrelation of CPI for $t \lesssim 12$ is out of the statistical fluctuations at the $95 \%$ level of confidence.
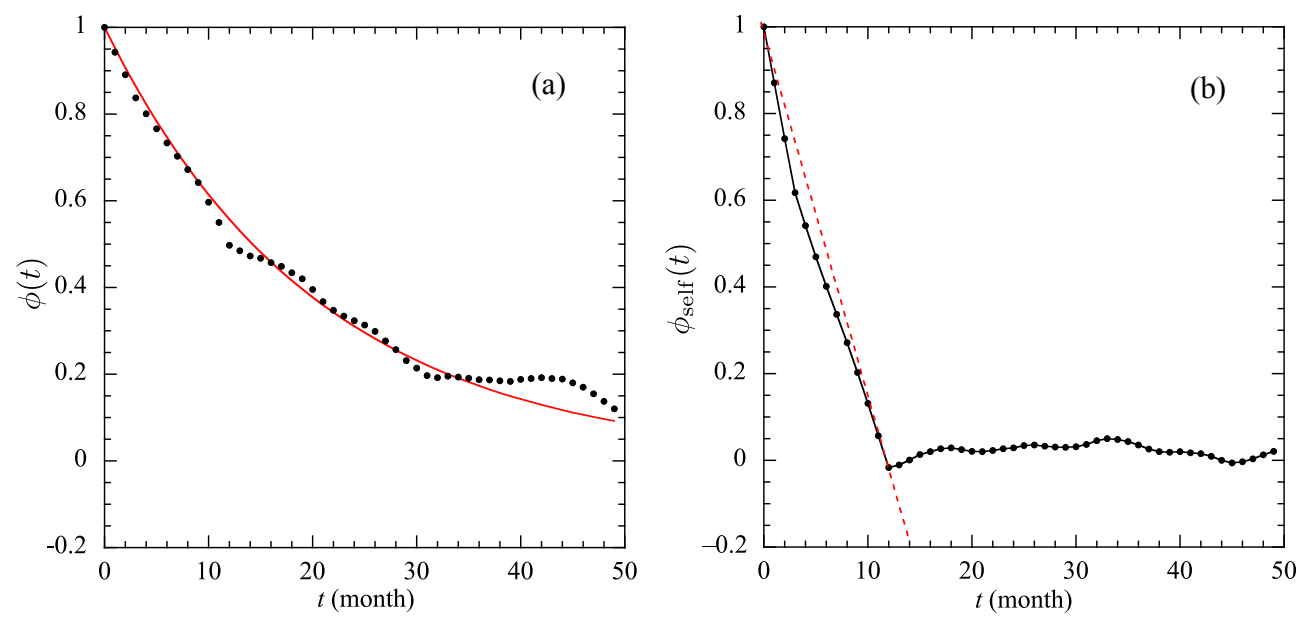

Figure 15. Same as Fig. 13 but for CPI with imputed rent excluded. The autocorrelation of the CPI decays exponentially with $\tau=20.5$.

change of aggregate price, namely deflation/inflation. This result implies that inertia is more important than expectations in the aggregate price dynamics because the standard assumption of rational expectations on the part of individual firms does not generate cross-autocorrelations of micro prices. In contrast, they naturally arise from input/output relationships in the production of goods and services; a rise of input price, for example, is shifted onto output price with a lag. We note that the 


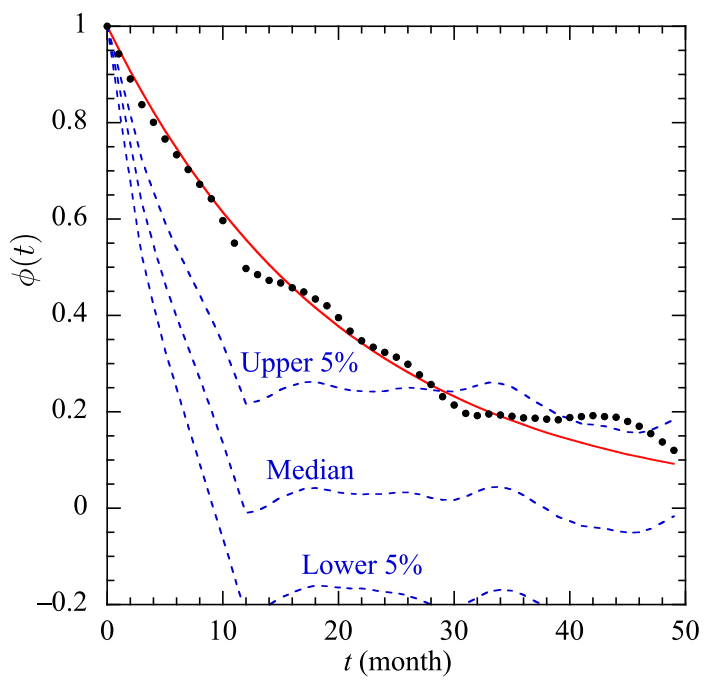

Figure 16. Same as Fig. 14 but for CPI with all items, less imputed rent. The characteristic decay time of its autocorrelation, measured by fitting to an exponential form, is 20.5 months. Interdependency of individual prices are more clearly visible here than in Fig. 14.

autocorrelations of individual prices (the first term of Eq. 10) are not significant after 12 months. It means that the menu costs which are to generate autocorrelations of individual prices are not really significant. The long autocorrelations of the aggregate price index arises mainly from cross autocorrelations of individual prices which cannot be generated by rational expectations. 


\section{Aggregate Price Index and Co-movements of Individual Prices}

Aggregate price index $P(t)$ is a weighted average of individual prices:

$$
P(t)=\sum_{\alpha=1}^{N} g_{\alpha} p_{\alpha}(t), \quad\left(\sum_{\alpha=1}^{N} g_{\alpha}=1\right) .
$$

Obviously, changes of aggregate price index $P(t)$ are caused by movements of in-

dividual prices. However, we know that an isolated change of price of particular good or service does not produce any significant change of aggregate price, namely deflation or inflation. At the same time, we recognize that prices of some products such as foodstuff and energy produce "noises" for the purpose of macroeconomics and monetary policy. That is why central banks target at the "core" CPI rather than CPI itself. However, the "core" CPI" is defined merely on common-sense and casual observation. In this section, based on the analysis of micro prices, we provide a method for defining the "systemic" changes in the aggregate price. It defines a kind of "true core" price.

Hereafter, we examine the rate of the change of price of good/service $\alpha, r_{\alpha}(t)$ defined as follows $(\alpha=1,2, \cdots, 830)$ :

$$
r_{\alpha}(t):=\log _{10}\left[\frac{p_{\alpha}(t+1)}{p_{\alpha}(t)}\right],
$$

where $t$ runs from 1 to $401(:=T)$. Then, the rate of the change of the aggregate price index $\Delta P(t) / P(t)$ where $\Delta P(t):=P(t+1)-P(t)$ can be expressed as follows:

$$
\begin{aligned}
\frac{\Delta P(t)}{P(t)} & =\sum_{\alpha=1}^{N} g_{\alpha} \frac{\Delta p_{\alpha}(t)}{P(t)} \\
& =\sum_{\alpha=1}^{N} g_{\alpha} \frac{p_{\alpha}(t)}{P(t)}\left(10^{r_{\alpha}(t)}-1\right) \\
& =\sum_{\alpha=1}^{N} g_{\alpha} \frac{p_{\alpha}(t)}{P(t)}\left(10^{\left\langle r_{\alpha}\right\rangle_{t}+\sigma_{\alpha} w_{\alpha}(t)}-1\right) \\
& \simeq c \sum_{\alpha=1}^{N} g_{\alpha} \frac{p_{\alpha}(t)}{P(t)}\left[\left\langle r_{\alpha}\right\rangle_{t}+\sigma_{\alpha} w_{\alpha}(t)\right],
\end{aligned}
$$

where $c:=\ln 10 \simeq 2.30$. Here, $\left\langle r_{\alpha}\right\rangle_{t}, \sigma_{\alpha}$ are the mean and the standard deviation of $r_{\alpha}(t)$. The normalized price change $w_{\alpha}(t)$ is defined by Eq. (2). We assumed that $\left\langle r_{\alpha}\right\rangle_{t} \ll 1$ and $\sigma_{\alpha} w_{\alpha}(t) \ll 1$ in the above. In what follows, we analyze $w_{\alpha}(t)$ rather than $r_{\alpha}(t)$ itself.

In order to analyze the co-movements of the individual prices of goods and services with lead-lag relations, we use the Complex Principal Component Analysis (CPCA). The ordinary principal component analysis (PCA) or factor analysis is meant to uncover "hidden" factors which generate co-movements of multi variables. 
Though it is widely used in economics as well as in other disciplines, it fails when movements of variables involve lead and lag relationships. This can be understood easily if one recalls a simple fact that the correlation of sine and cosine curves becomes zero; in this example, there is a systematic relation between two variables, and yet, the presence of leads/lags makes the correlation zero (see Appendix A). We can explicitly take into account leads and lags present in micro price dynamics by using $\mathrm{CPCA}$.

\section{Complex Principal Component Analysis}

The CPCA consists of the following steps;

A. We construct complex time-series by adding each time-series the Hilbert transform of the original time-series as the imaginary part; for the Hilbert transformation see Granger and Hatanaka (1964).

B. We then calculate the matrix of the correlation coefficients of constructed complex time series, its eigenvalues, and eigenvectors.

C. In order to separate significant eigenmodes that represent "true" co-movements (signals) from the noise eigenmodes, we carry out the significance test by Rotation Random Shuffling (RRS) simulations.

We explain three steps below.

\section{The Hilbert Transformation and the Complexified Time-series}

The discrete Fourier expansion of a time-series $r(t)(t=1,2, \cdots T)$ is as follows:

$$
r(t)=\frac{1}{\sqrt{T}} \sum_{k=1}^{T} r^{(\mathrm{F})}(k) e^{-i \frac{2 \pi}{T} k t}, \quad r^{(\mathrm{F})}(k)=\frac{1}{\sqrt{T}} \sum_{t=1}^{T} r(t) e^{i \frac{2 \pi}{T} k t} .
$$

Because $r(t)$ is real, $r^{(\mathrm{F})}(k)=r^{(\mathrm{F})}(T-k)^{*}$ and $r^{(\mathrm{F})}(T)=\sum_{m=1}^{T} r(t) / \sqrt{T}$ is real. For even $T$, the Fourier expansion is written as follows,

$$
r(t)=\frac{1}{T} \sum_{t^{\prime}=1}^{T}\left(1+(-1)^{t+t^{\prime}}\right) r\left(t^{\prime}\right)+\frac{2}{\sqrt{T}} \Re\left[\sum_{k=1}^{T / 2-1} r^{(\mathrm{F})}(k) e^{-i \frac{2 \pi}{T} k t}\right],
$$

where $\Re$ denotes the real part (and we use $\Im$ for imaginary part later). The 1 st term in the left-hand side comes from $k=T, T / 2$ terms.

\footnotetext{
${ }^{6}$ These are described by some of the current authors in Vodenska et al. (2014). We, however, will review them in the following for completeness and convenience of the readers.
} 
The Hilbert transform creates the imaginary part corresponding to the second term on the right-hand side of the above equation:

$$
r^{(\mathrm{H})}(t):=\frac{2}{\sqrt{T}} \Im\left[\sum_{k=1}^{T / 2-1} r^{(\mathrm{F})}(k) e^{-i \frac{2 \pi}{T} k t}\right] .
$$

By adding this to the original time-series as the imaginary part, we obtain complexified time-series $\widetilde{r}(t)$ as follows:

$$
\widetilde{r}(t):=r(t)+i r^{(\mathrm{H})}(t)=\frac{1}{T} \sum_{t^{\prime}=1}^{T}\left(1+(-1)^{t+t^{\prime}}\right) r\left(t^{\prime}\right)+\frac{2}{\sqrt{T}} \sum_{k=1}^{T / 2-1} r^{(\mathrm{F})}(k) e^{-i \frac{2 \pi}{T} k t} .
$$

Similarly, we have the following equations for odd $T$ :

$$
\begin{aligned}
& r(t)=\frac{1}{T} \sum_{t^{\prime}=1}^{T} r\left(t^{\prime}\right)+\frac{2}{\sqrt{T}} \Re\left[\sum_{k=1}^{(T-1) / 2} r^{(\mathrm{F})}(k) e^{-i \frac{2 \pi}{T} k t}\right], \\
& \widetilde{r}(t):=\frac{1}{T} \sum_{t^{\prime}=1}^{T} r\left(t^{\prime}\right)+\frac{2}{\sqrt{T}} \sum_{k=1}^{(T-1) / 2} r^{(\mathrm{F})}(k) e^{-i \frac{2 \pi}{T} k t} .
\end{aligned}
$$

We note that both Eq. (18) and (20) rotate clockwise in the complex plane.

\section{Complex Correlation Matrix}

The normalized rate of change $\widetilde{w}_{\alpha}$ corresponding to the complexified rate of change of individual price $\widetilde{r}_{\alpha}(t)$ is defined by

$$
\widetilde{w}_{\alpha}(t):=\frac{\widetilde{r}_{\alpha}(t)-\left\langle\widetilde{r}_{\alpha}\right\rangle_{t}}{\sigma_{\alpha}}
$$

where $\langle\cdot\rangle_{t}$ denotes the average over time $t=1, \ldots, T\left(\langle\cdot\rangle_{t}:=(1 / T) \sum_{t=1}^{T} \cdot\right)$, and $\sigma_{\alpha}(\geq 0)$ denotes the standard deviation of $\widetilde{r}_{\alpha}$ over time;

$$
\sigma_{\alpha}^{2}:=\frac{1}{T} \sum_{t=1}^{T}\left|\widetilde{r}_{\alpha}(t)-\left\langle\widetilde{r}_{\alpha}\right\rangle_{t}\right|^{2}=\sum_{t=1}^{T}\left\langle\left|\widetilde{r}_{\alpha}(t)\right|^{2}\right\rangle_{t}-\left|\left\langle\widetilde{r}_{\alpha}\right\rangle_{t}\right|^{2}
$$

The complex correlation matrix $\widetilde{\boldsymbol{C}}$ is the $N \times N(N=830)$ matrix with its components defined as follows;

$$
\widetilde{C}_{\alpha \beta}:=\left\langle\widetilde{w}_{\alpha} \widetilde{w}_{\beta}^{*}\right\rangle_{t}
$$

where $\widetilde{w}_{\beta}^{*}$ is complex conjugate to $\widetilde{w}_{\beta}$. This matrix is Hermitian by construction:

$$
\widetilde{\boldsymbol{C}}^{\dagger}=\widetilde{\boldsymbol{C}} \quad\left(\widetilde{C}_{\alpha \beta}^{*}=\widetilde{C}_{\beta \alpha}\right) .
$$


The diagonal elements of the matrix $\widetilde{\boldsymbol{C}}$ are 1 by definition of the normalized growth rate $\widetilde{w}_{\alpha}$.

The phase of the complex correlation coefficient (23) corresponds to the lead-lag relationship between the time-series $\alpha$ and $\beta$ : For odd $T$, which is our case, the normalized logarithmic rate of change $\widetilde{w}_{\alpha}$ is expanded as follows:

$$
\widetilde{w}_{\alpha}(t)=\frac{2}{\sqrt{T}} \sum_{k=1}^{(T-1) / 2} w^{(\mathrm{F})}(k) e^{-i \frac{2 \pi}{T} k t}=\sum_{k=1}^{(T-1) / 2}\left|w^{(\mathrm{F})}(k)\right| e^{i\left(\delta_{\alpha}(k)-\frac{2 \pi}{T} k t\right)},
$$

where $\delta_{\alpha}(k)$ denotes the phase of $w_{\alpha}^{(\mathrm{F})}(k)$. Substituting Eq.25 into Eq. 23, we find that

$$
\widetilde{C}_{\alpha \beta}:=\frac{4}{T} \sum_{k=1}^{(T-1) / 2} w_{\alpha}^{(\mathrm{F})}(k) w_{\beta}^{(\mathrm{F})}(k)^{*}=\frac{4}{T} \sum_{k=1}^{(T-1) / 2}\left|w_{\alpha}^{(\mathrm{F})}(k) w_{\beta}^{(\mathrm{F})}(k)^{*}\right| e^{i\left(\delta_{\alpha}(k)-\delta_{\beta}(k)\right)} .
$$

This means that the phase of the complex correlation coefficient $\widetilde{C}_{\alpha \beta}$ represents how the time-series $\alpha$ lags behind the time-series $\beta$ : If there is only one Fourier-component of $k=k_{0}$ in each of the time-series, the phase of the complex correlation coefficient $\widetilde{C}_{\alpha \beta}$ is equal to $\delta_{\alpha}\left(k_{0}\right)-\delta_{\beta}\left(k_{0}\right)$. Since their period is $T / k_{0}$, this means that the time series $\alpha$ lags behind the time-series $\beta$ by the time-difference $\left(\delta_{\alpha}\left(k_{0}\right)-\delta_{\beta}\left(k_{0}\right)\right) T /\left(2 \pi k_{0}\right)$ (see Fig.17). If there are multiple Fourier components in any of the time-series $\alpha$ and $\beta$, the phase of the complex correlation coefficient $\widetilde{C}_{\alpha \beta}$ is a weighted (non-linear) average of the time-delay as in Eq.26). For even $T$, the similar relation holds.

One may think that the lead-lag relation can be investigated using the traditional correlation analysis by time-shifting time-series relative to each other, and obtaining the best estimate of the time-delay by maximizing the correlation coefficient. While this has an advantage of having explicit lead-time, it becomes almost impossible to calculate for multiple time-series: We have $N=830$ time series and if we allow them to shift by, say, 6 months for each pair, the number of coefficients to calculate is of order of $6 N(6 N-1) / 2 \simeq 12.5 \times 10^{6}$. Optimization is practically impossible. Compared with such calculation, our analysis has a substantial advantage of having just one correlation matrix.

The eigenvalues $\lambda^{(n)}$ and the eigenvectors $\boldsymbol{V}^{(n)}$ are as follows:

$$
\begin{aligned}
& \widetilde{\boldsymbol{C}} \boldsymbol{V}^{(n)}=\lambda^{(n)} \boldsymbol{V}^{(n)}, \\
& \boldsymbol{V}^{(n) \dagger} \cdot \boldsymbol{V}^{(m)}=\delta_{n m} .
\end{aligned}
$$

They satisfy the following relations:

$$
\begin{aligned}
& \sum_{n=1}^{N} \lambda^{(n)}=N \\
& \widetilde{C}=\sum_{n=1}^{N} \lambda^{(n)} \boldsymbol{V}^{(n)} \boldsymbol{V}^{(n) \dagger} .
\end{aligned}
$$




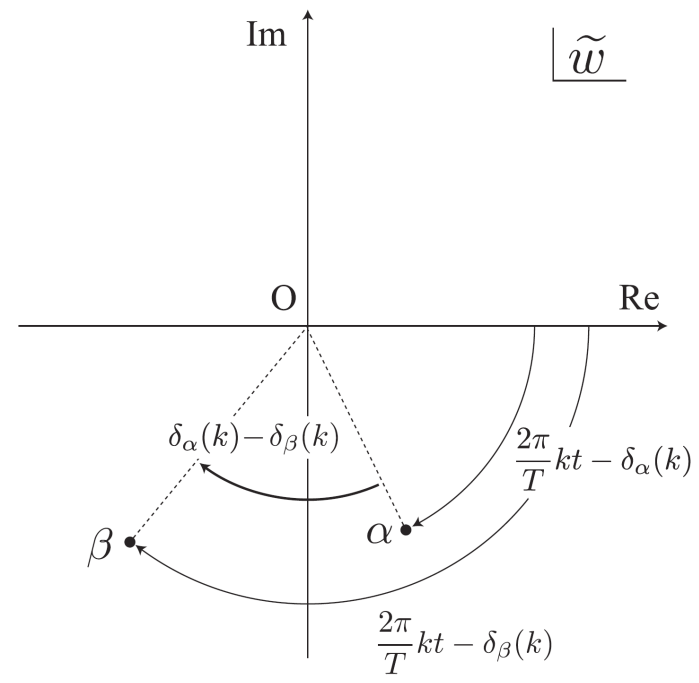

Figure 17. Lead-lag relationship expressed by the CPCA correlation coefficient $\widetilde{\boldsymbol{C}}_{\alpha \beta}$ for a given $k$ in Eq. 26.

The eigenvalues of conventional (real) correlation matrix with real components are non-negative because of its chiral nature. This mathematical property remains intact for the correlation matrix $\widetilde{C}$ with complex components. Namely,

$$
\lambda^{(n)}=\left\langle\left|\sum_{\alpha} \widetilde{w}_{\alpha}^{*}(t) V_{\alpha}^{(n)}\right|^{2}\right\rangle_{t} \geq 0,
$$

which is derived by multiplying both sides of Eq. (27) by $V^{(n) \dagger}$ from the left with substitution of Eq. 23 into $\widetilde{C}]^{7}$

The normalized rate of change of individual price, $\widetilde{w}_{\alpha}$ defined by Eq. 221) can be

\footnotetext{
${ }^{7}$ Let us make a comment on the number of zero modes. The matrix $\widetilde{C}$ is a non-regular matrix with rank $T / 2-1$ for even $T$ or $(T-1) / 2$ for odd $T$ in the present case of $N>T$, leading to the existence of $N-T / 2+1$ or $N-(T-1) / 2$ trivial zero eigenvalues, respectively. This is appreciated through an explicit expression for the $\beta$-th column vector $\widetilde{\boldsymbol{c}}_{\beta}$ of $\widetilde{C}$ given as

$$
\widetilde{\boldsymbol{c}}_{\beta}=\frac{1}{T} \sum_{t=1}^{T} \widetilde{w}_{\beta}(t) \widetilde{\boldsymbol{w}}(t),
$$

where $\widetilde{\boldsymbol{w}}(t)$ is a column vector representation of the normalized rates of change $\left\{\widetilde{w}_{\alpha}(t)\right\}$ at time $t$. Equations $(18)$ and 20 however show that all of $T \widetilde{\boldsymbol{w}}(t)$ 's are not mutually independent. The numbers of independent terms on the right hand of Eqs. $(18)$ and $(20)$ are just $T / 2+1$ and $(T+1) / 2$, respectively. The standardization of data, Eq. 21), imposes one more constraint on the column vectors; the sum of them exactly vanishes: $\sum_{t} \widetilde{\boldsymbol{w}}(t)=0$. We thus see that only $T / 2-1$ or $(T-1) / 2$ column vectors of $\widetilde{C}$ are independent among the totally $N$ column vectors depending on whether $N$ is even or odd.
} 
expanded in terms of these eigenvectors,

$$
\widetilde{w}_{\alpha}(t)=\sum_{n=1}^{N} a^{(n)}(t) \boldsymbol{V}_{\alpha}^{(n)} .
$$

We call the coefficients $a^{(n)}(t)$ mode-signals. Using Eqs. 23, 27), and (28), we see that

$$
\left\langle a^{(n) *} a^{(m)}\right\rangle_{t}=\delta_{n m} \lambda^{(n)} .
$$

This means the following:

1. The mode-signals are independent from each other (as they should, belonging to independent eigenvectors).

2. The larger the eigenvalue is, the larger the eigenvector's presence is. More accurately, their mean strength is proportional to the square root of the eigenvalues.

\section{Rotational Random Shuffling (RRS) Method: A Significance Test}

We must study which eigenmodes are significant, i.e., signals representing systemic co-movements in this system, not noises. It is the central issue that we always encounter in applying PCA to multivariate data.

The random matrix theory (RMT) provides us with a sound null hypothesis for such a statistical significance test. A set of random iid (independent, identically distributed) time-series has a non-trivial correlation matrix and the eigenvalue spectrum $\rho(\lambda)$ is explicitly calculated as

$$
\begin{gathered}
\rho(\lambda)=\frac{Q}{2 \pi} \frac{\sqrt{\left(\lambda_{+}-\lambda\right)\left(\lambda-\lambda_{-}\right)}}{\lambda}, \\
\lambda_{ \pm}=\left(1 \pm \frac{1}{\sqrt{Q}}\right)^{2} .
\end{gathered}
$$

where $Q=T / N$ and $\left.\lambda_{-} \leq \lambda \leq \lambda_{+}\right]^{8}$ Because the eigenvalues predicted by RMT are confined in $\left[\lambda_{-}, \lambda_{+}\right]$, the eigenvalues for the actual correlation matrix larger than $\lambda_{+}$ can be regarded as representing statistically meaningful correlations.

\footnotetext{
${ }^{8}$ This formula was first derived by Marčenko and Pastur (1967). If $Q<1$, we have to add a contribution of eigenvalues condensed at zero with fraction $1-Q$ to the right-hand side of Eq. (34). For the CPCA, $Q$ in Eqs. (34) and (35) should be replaced by $Q / 2$ as has been noted by Arai and Iyetomi (2013); this is because the imaginary part of complexified time series is not independent of its real part, related through the Hilbert transformation. RMT is a refinement of Kaiser's selection rule, $\lambda>1$. One can further improve it by comparing the actual eigenvalues with the corresponding eigenvalues of RMT rank by rank, in place of $\lambda_{+}$. This method is basically the same as the one originally proposed by Horn (1965), known as parallel analysis (PA) in statistics (Zwick and Velicer (1986); Buja and Eyuboglu (1992); Franklin et al. (1995)). However, PA does not take advantage of RMT; instead it carries out numerical simulations for its null model.
} 
While the RMT-based method is clearly superior to other methods from both theoretical and practical points of view, Iyetomi et al. (2011) demonstrates that it requires the following to be satisfied: 1 ) there is no autocorrelation in each timeseries, and 2) the time-series are infinite in the sense that $N, T \rightarrow \infty$ with $Q=T / N$ kept finite.

To be free of the above restrictions on the applicability of RMT to PCA, we resort to RRS simulation proposed in Arai and Iyetomi (2013). In this simulation, we first randomly rotate each time-series as follows;

$$
\widetilde{w}_{\alpha}(j) \rightarrow \widetilde{w}_{\alpha}\left(\operatorname{Mod}\left(j-\tau_{\alpha}, T\right)+1\right)
$$

where $\tau_{\alpha} \in[0, N]$ and $\operatorname{Mod}(n, m)$ is the modulus function to give the remainder of division of $n$ by $m$. It should be noted here that no auto-correlation is lost in each time-series, as they are "rotated", which are necessary for keeping the length of the time-series intact. On the other hand, since each time-series is rotated differently, the comovement between them are destroyed. Therefore, the resulting eigenvalues $\lambda^{(n)}$ should reflect the same set of time-series with co-movements destroyed. This in turn means that by comparing the resulting eigenvalue spectrum with the actual one we can identify what are true co-movements in the data.

\section{The Results}

We have applied the method explained above to our micro price data. Fig 18 is the comparison of the actual eigenvalues and the RRS results. The gray area is obtained by carrying out the random rotation $10^{3}$ times and excluding lower 5 and upper 5 eigenvalues. Applying the parallel analysis mentioned in the previous subsection, we can conclude that the first 26 eigenmodes are significant; they are outside of the range of the $99 \%$ RRS results. The significant 26 eigenmodes represent "true" systemic co-movements of individual prices.

The green curve in Fig. 19 shows the cumulative value of the eigenvalues

$$
S_{n}:=\sum_{k=1}^{n} \lambda^{(k)}
$$

on the ordinate and $n$ on the abscissa. As explained previously, we have $N-(T-$ $1) / 2=630$ (as $N=830, T=401$ ) zero eigenvalues in CPCA results, the green curve reaches the total value 830 at $n=200 ; S_{200}=830$. The black vertical line is at $n=26$, where $S_{26}=397.45$. Since the mode signal's presence in the time series is governed by its eigenvalue as in Eq. (33), this means that that the 26 significant modes cover $S_{26} / 830=0.48$, or $48 \%$ of all the time-series behavior. The blue curve in this plot is the corresponding result for PCA. This shows that CPCA eigenmodes corresponds to stronger correlation than that of PCA consistently through all the modes. 


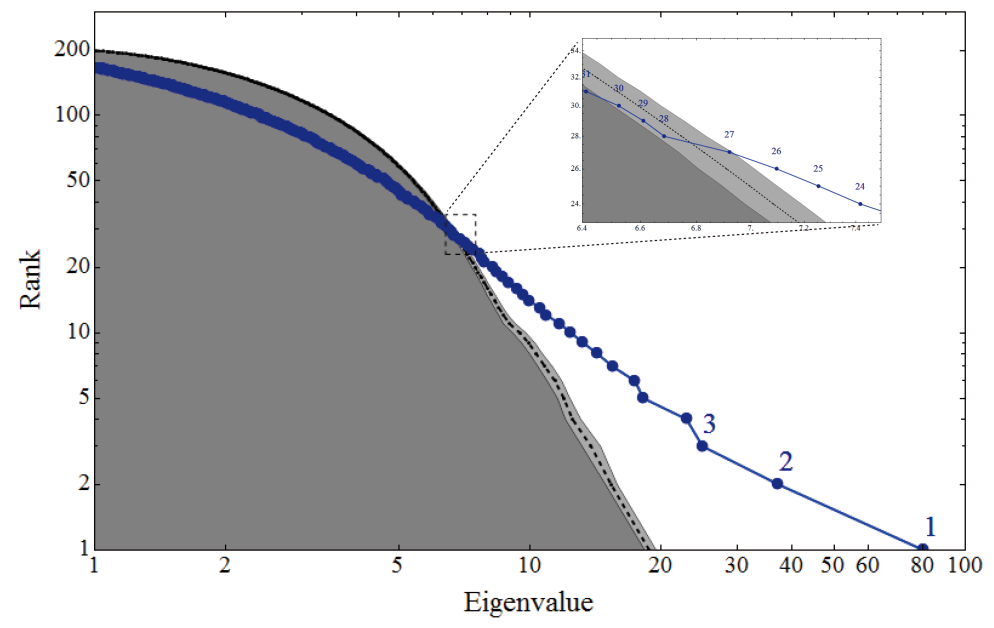

Figure 18. Eigenvalues obtained by the CPCA and the RRS results with abscissa showing the eigenvalues and the ordinate the rank in the descending order. The blue dots connected with blue lines are the actual CPCA eigenvalues with those denoted " $n$ " (for $n=1,2,3$ ) corresponding to the $n$-th largest eigenvalue. The gray small dots and the light gray area show the average RRS and the $99 \%$ range. The inset shows the detail of the main plot where CPCA eigenvalues cross under the RRS range, from which we find that the largest 26 eigenvalues are clearly outside of their RRS ranges, and are identified as signals of the the co-movements in this system.

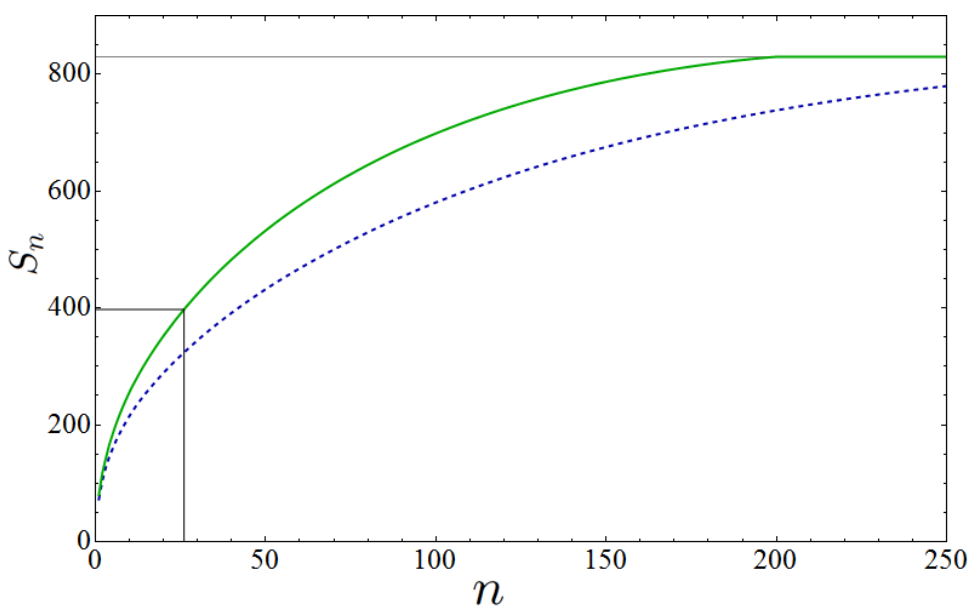

Figure 19. The cumulative eigenvalues $S_{n}$ defined by Eq. (37) for CPCA (green solid line) and PCA (blue dashed line). We observe that 26 significant CPCA eigenmodes explain about $48 \%$ of all the behavior of the time-series. 


\section{Two Case Studies}

Once we obtain the significant eigenvectors, we can express the normalized rate of change of individual price by using only these eigenvectors,

$$
\widetilde{w}_{\alpha}(t)=\sum_{n=1}^{N_{\mathrm{s}}} a^{(n)}(t) \boldsymbol{V}_{\alpha}^{(n)} .
$$

where $N_{\mathrm{s}}=26$ is the number of significant modes. To see how the individual prices projected onto the 26 significant modes behave, we do two case studies. For two periods we examine, we compare the original time series Eq. 32 with Eq. 38. In each figure, we have 830 individual prices.

(a) Appreciation of the Yen following the Plaza Agreement, 1985-1988, revisited

Fig. 20 shows (a) the original time-series and (b) the one projected to the 26 significant eigenmodes, namely Eq. (38), for the period 1985-88 when the yen sharply appreciated from 240 to 120 per US dollar following the Plaza Agreement in September 1985. Plot (b) more clearly shows that import prices significantly fell during this

period than the original series in (a). This shows the power and the usefulness of our approach; since we have removed noises by way of projection to significant 26 modes, the resulting plot (b) is much clearer than the plot (a).

Figure 21 is the plot of the averaged values of the projected time-series for sectors listed in Table 1 rather than 830 micro prices. It is clear that many of import prices such as chemicals, petroleum and coal products, and nonferrous metals fell starting October 1985, and then DCGPI prices followed.

Fig. 22 shows DCGPI by sector. In some sectors, prices drastically change (left), whereas in others, no significant changes are observed (right). Fig. 23 is for the CPI. Here, we observe no significant changes.

Figure 24 shows the behavior of the projected series on its complex plane during this period. The major impulse to import prices is clearly seen. 


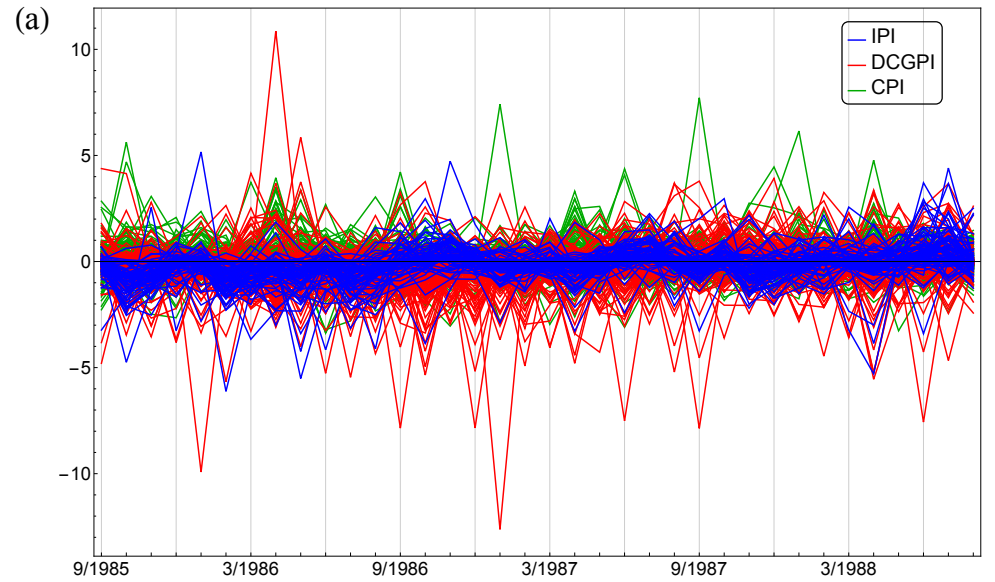

(b)

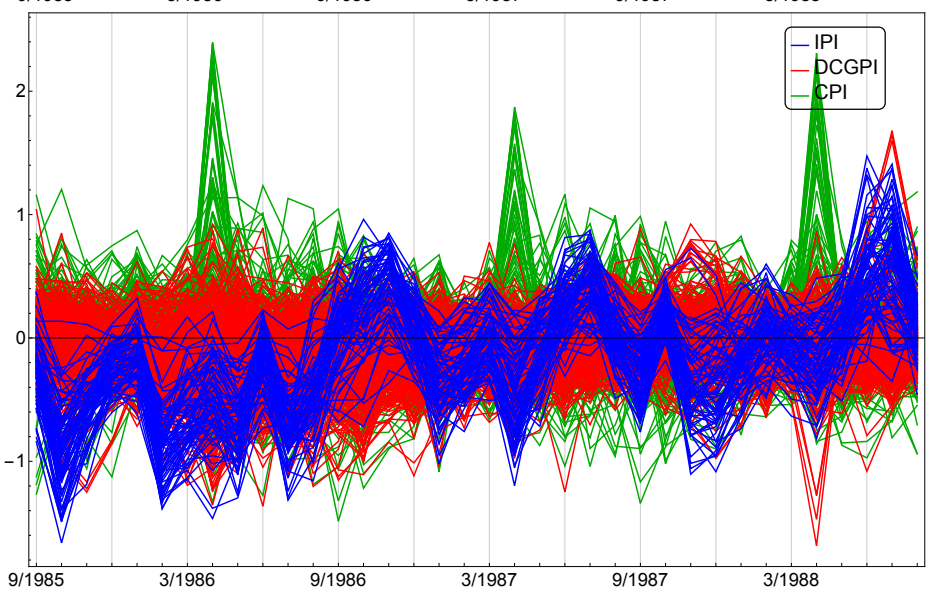

Figure 20. The real part of the time-series in and out of the period of Yen appreciation. Plot (a) shows the original time-series, while (b) shows the time-series projected to the 26 significant modes. (Note the difference in vertical scale.) It is apparent that the projected time-series shows distinct peak structures, while the original series, being contaminated with noises, does not.

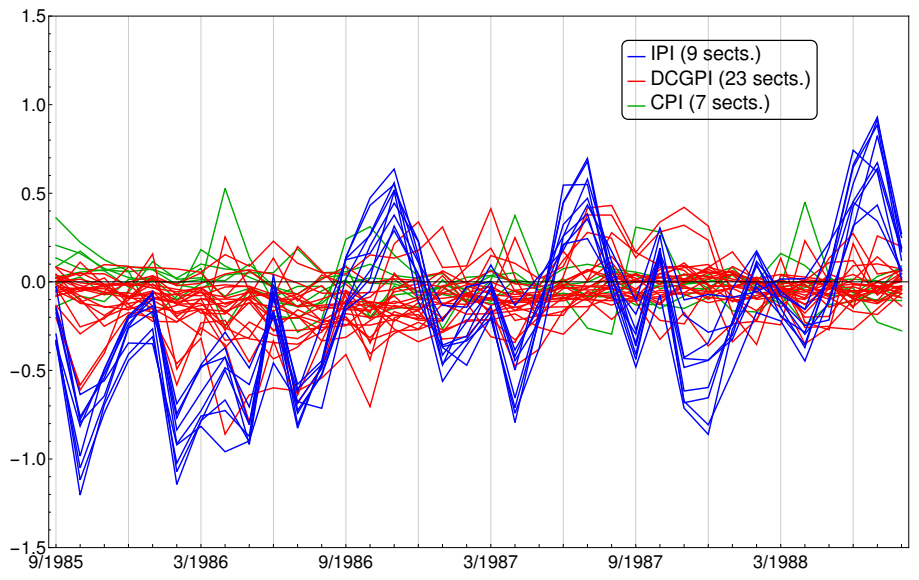

Figure 21. Plot of the averaged values of the projected data in each sectors. The reduction in IPI followed by DCGPI is much more distinct here than in previous figures. 

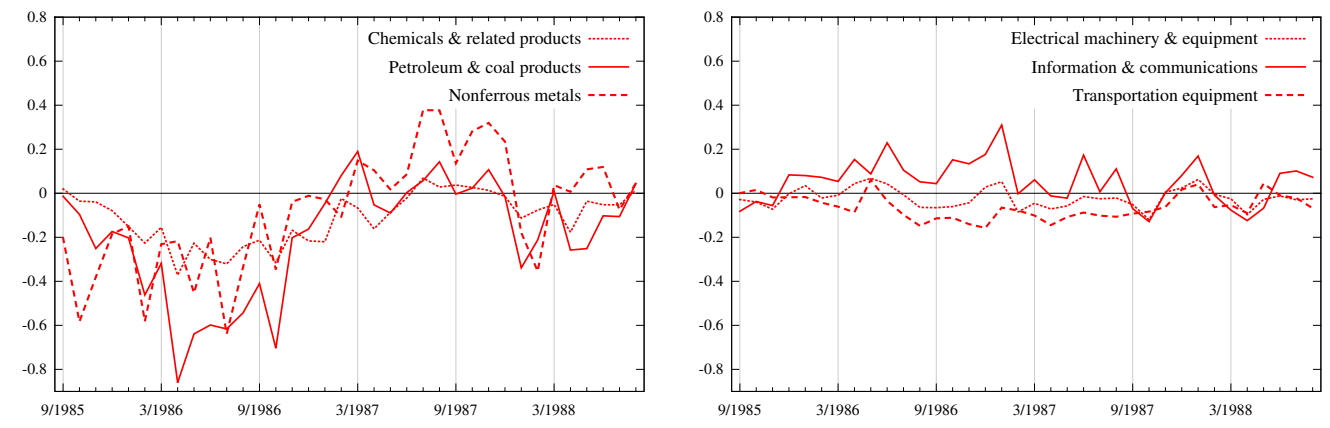

Figure 22. DCGPI sectors which showed significant price changes (left), and no significant changes (right).

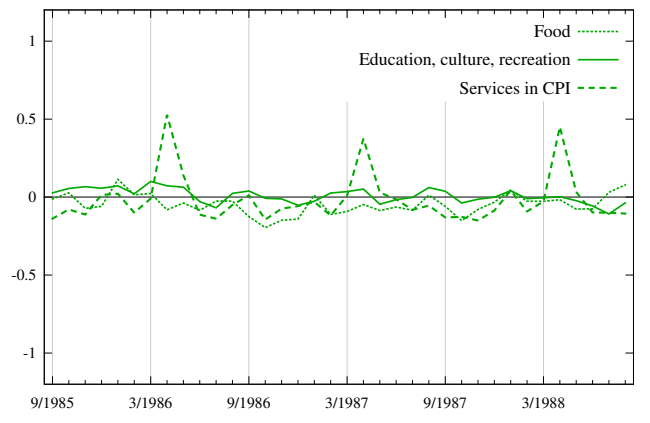

Figure 23. CPI by sector. No significant change is observed in any.

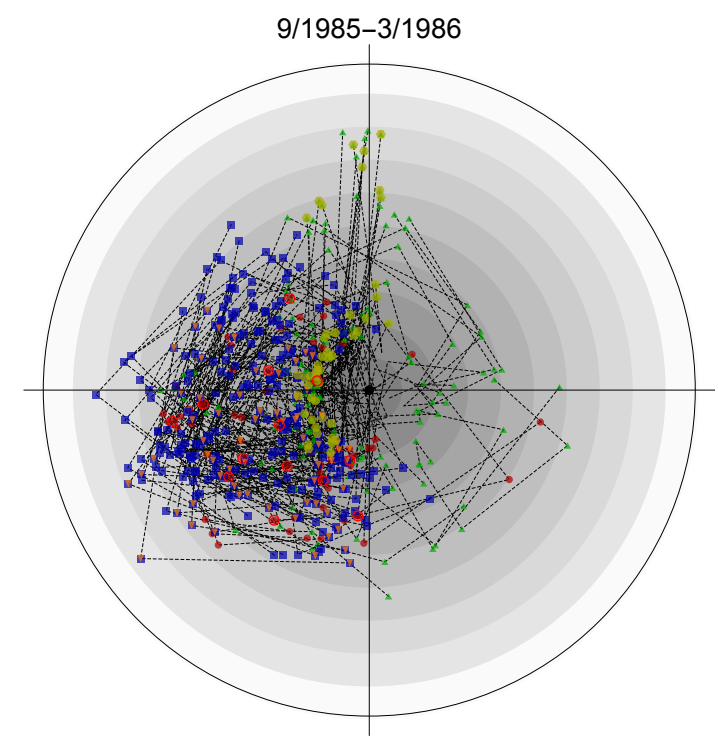

Figure 24. The behavior of the projected series on its complex plane during the period of Yen appreciation. The six markers of each micro price for this six month period are connected by dashed lines. Markers for points with radius less than 0.3 are not drawn to avoid crowding. 


\section{(b) The Great Recession, 2008-2009, revisited}

Fig. 25 shows the behavior of (a) the original time-series and (b) the one projected to the 26 significant eigenmodes for the Great Recession, 2008-09. We again observe that the projected time-series shown in (b) has clear and distinctive 5 peaks starting in April 2008. We note that these peaks are not clearly visible in the original timeseries. Furthermore, the projected series in (b) removes the fluctuations before March 2008 and after May 2009, particularly in DCGPI observed in the original series. The difference arises, of course, from the noises we have removed from the original timeseries. Fig. 26 shows the behavior of sectors comprising DCGPI rather than 830 micro prices. The significant increases of some prices before September, 2008 and the decreases of these same prices after the bankruptcy of the Lehman Brothers in September 2008 is most clearly observed.

Fig. 27 shows the behavior of some sectors of DCGPI prices which show drastic changes (left) and others which show basically no changes (right). Fig. 28 is CPI by sector. Prices of chemicals, petroleum and coal products, and iron and steel in DCGPI significantly rose before September 2008, and then fell afterwords. The similar pattern is observed for food and automobile prices in CPI albeit to lesser extent. On the other hand, prices of production machinery, electric machinery, and transportation equipment in DCGPI, and prices of education/culture/recreation and services in CPI all showed only minor fluctuations during the period.

The behavior of the complex time-series for three month period at 5 peaks observed in Fig. 25 (b) is plotted in Fig. 29. The first peak of micro prices is April 2008, which is observable from the fact that several DCGPI prices are on the far right hand side of the circle, meaning that their real part is positive and large. Most of these are construction-related prices (marked by red circle), including raw materials such as iron ore, steel, quicklime and coal, and products like wire, welding rods, and ceramic cladding. They reflect strong real economic activities, construction in particular. Others notable for big positive change are raw milk and milk products such as butter and cheese. They reflected rises of global prices of some foodstuffs during the period. 

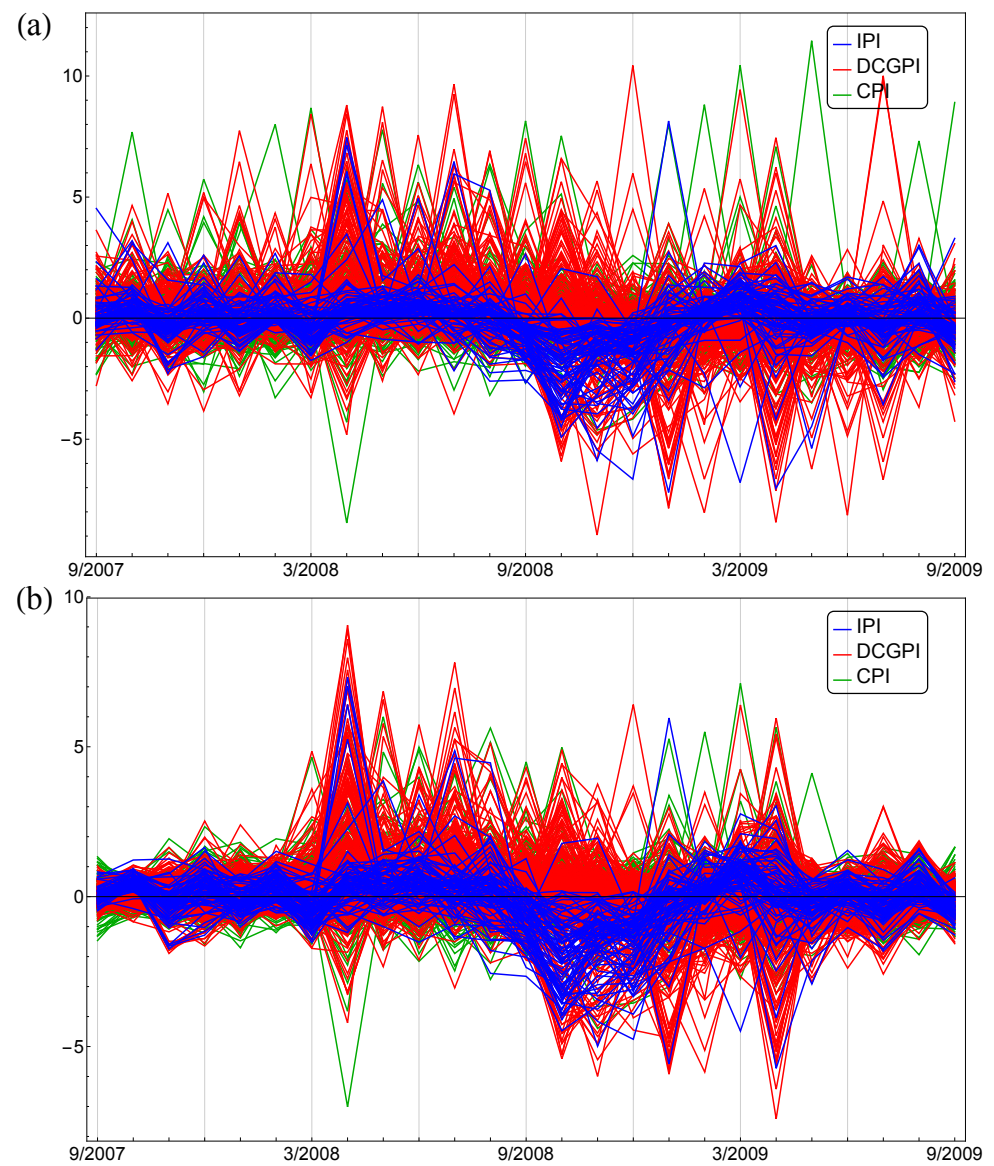

Figure 25. The real part of the time-series in and out of the Great Recession period. Plot (a) shows the original time-series, while (b) shows the time-series projected to the 26 significant modes. It is apparent that the projected time-series shows distinct peak structures, while the original series, being contaminated with noises, does not.

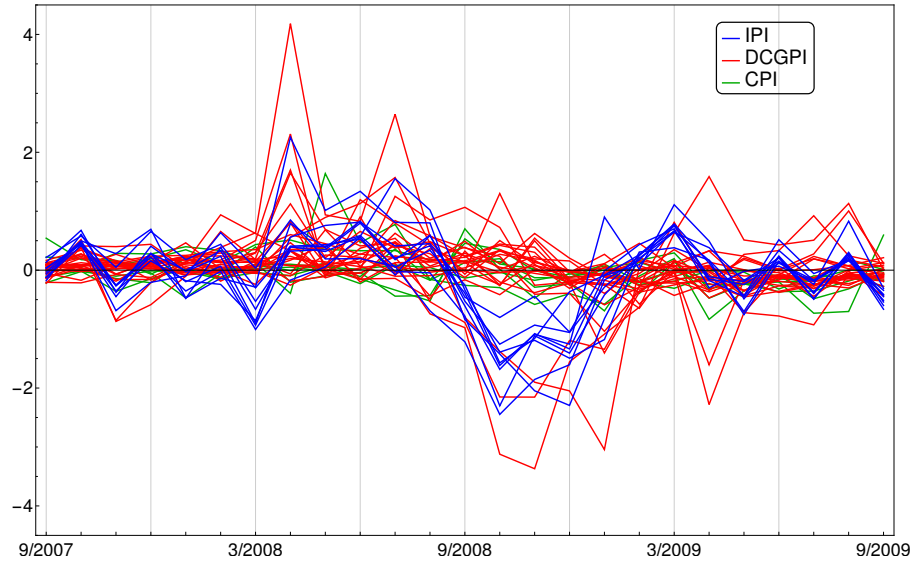

Figure 26. Plot of the averaged values of the projected data in each sectors. 

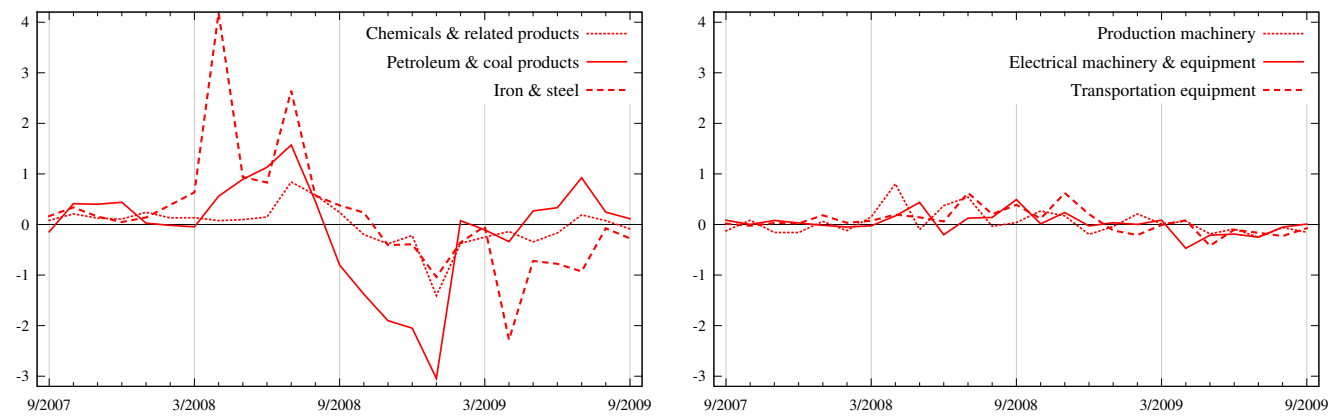

Figure 27. The behaviors of the DCGPI sectors with drastic changes (left) and no much change (right).
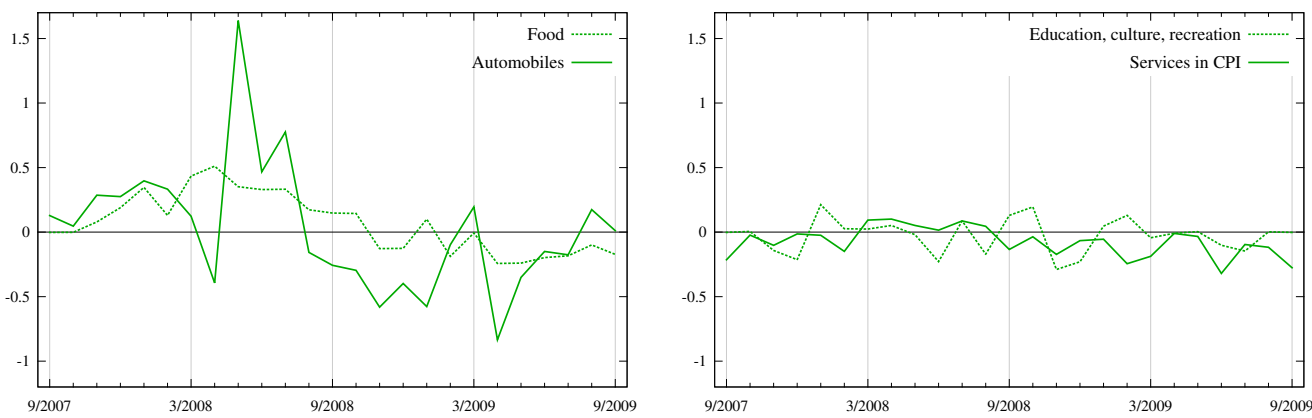

Figure 28. The behaviors of the CPI sectors with drastic changes (left) and no much change (right). 

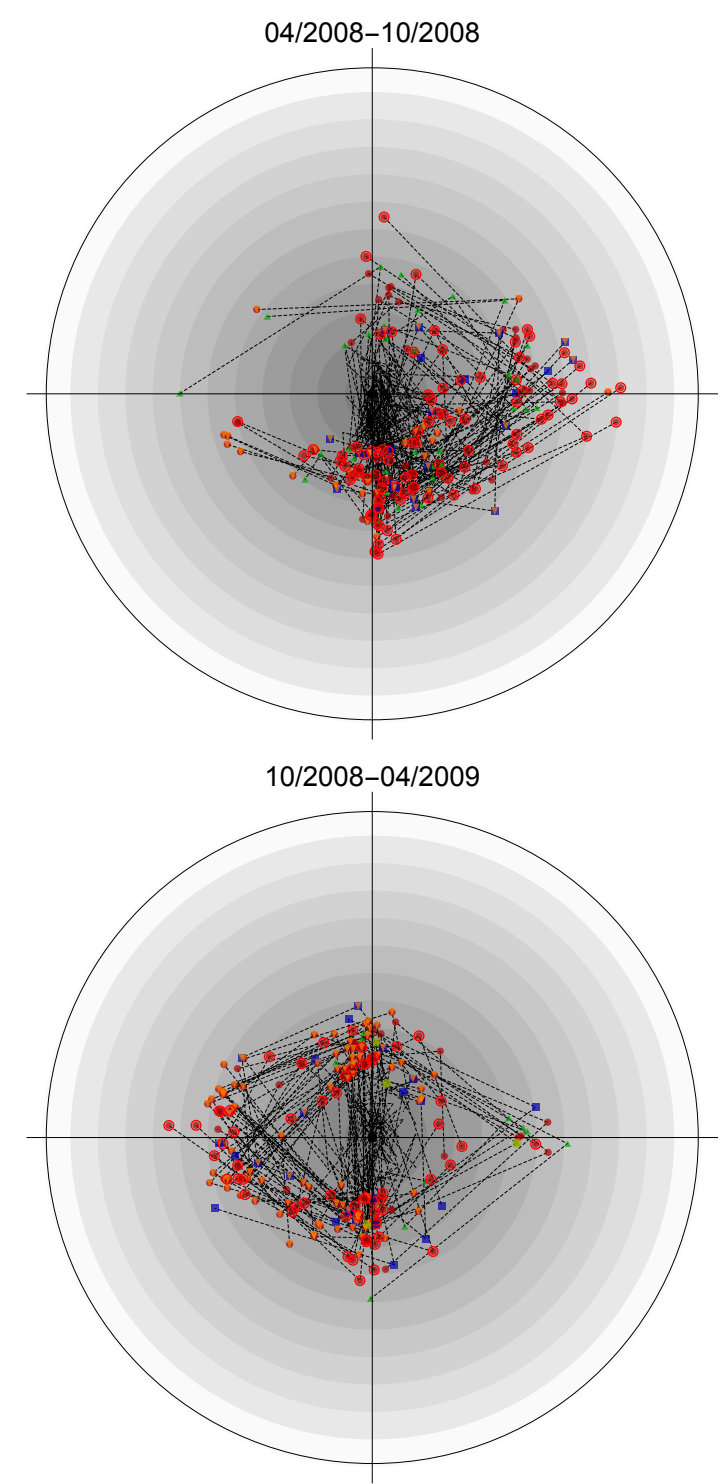

Figure 29. The projected time-series in their complex plane for the first six months from April to October 2008 and the second six months from October 2008 to April 2009. For each micro price, the values of the six months are connected by dashed lines. Plot markers are not drawn for points with radius less than 2 . 


\section{Properties of Leading Eigenvectors}

We examine the properties of some of the most important eigenvectors. Fig 30 is the plot of the components of the first eigenvector, namely, $V_{\alpha}^{(1)}$ for $\alpha=1,2, \cdots 830$ in the complex plane. Since the phase of the eigenvector is arbitrary, we chose the phase of the eigenvector in such a way that the spread of the components are the largest along the positive real axis, that is

$$
\Re\left(\boldsymbol{V}^{(1)}\right) \cdot \Im\left(\boldsymbol{V}^{(1)}\right)=0, \quad\left|\Re\left(\boldsymbol{V}^{(1)}\right)\right| \geq\left|\Im\left(\boldsymbol{V}^{(1)}\right)\right| .
$$

The plot marks are separately given for 3 major categories, IPI, DCGPI, and CPI, and overlays are given for sub-categories "Oil-related Goods", "Services", and "Constructionrelated good", as shown in the legend.

CPCA Eigenvector No.1

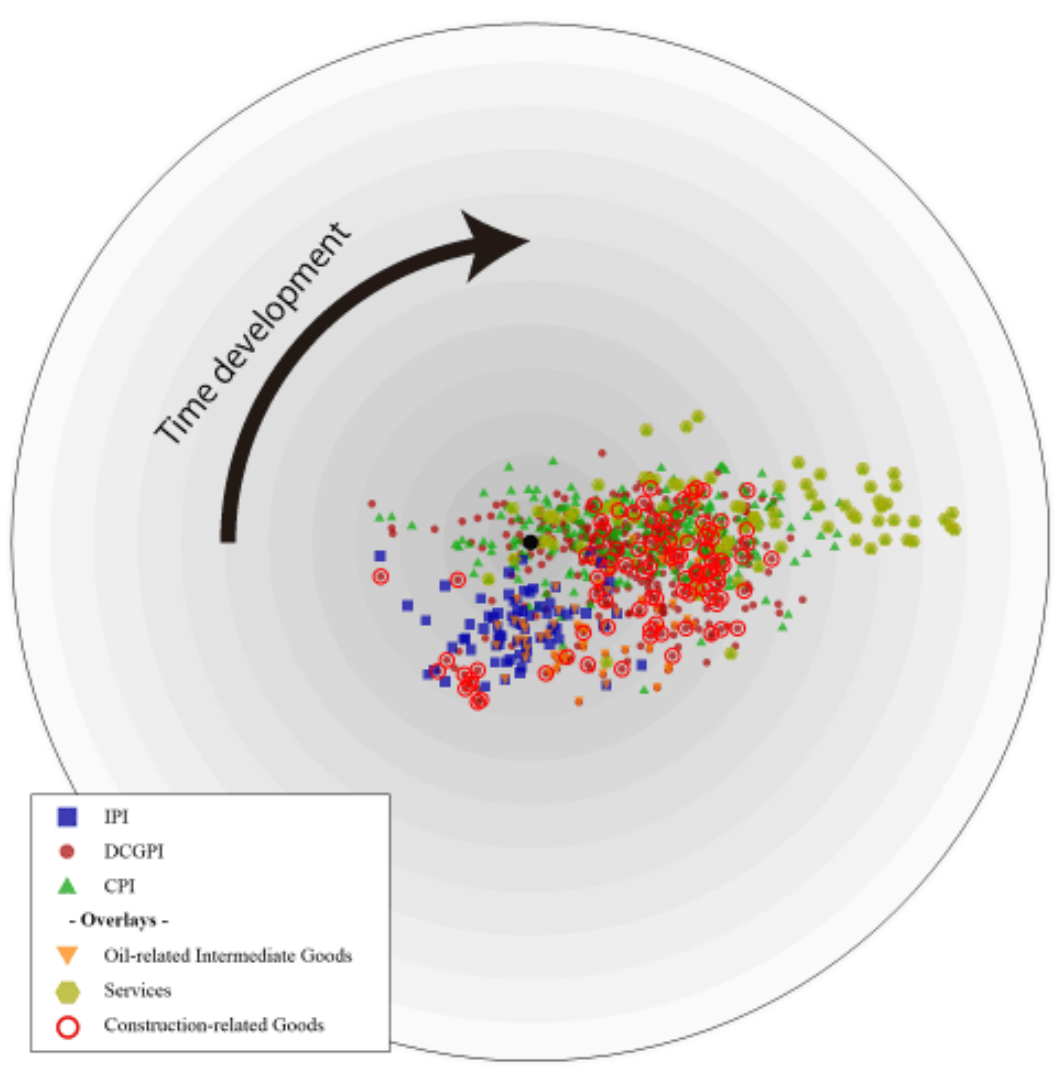

Figure 30. The 1st eigenvector components in the complex plane.

To study the characteristics of this eigenvector, we first calculate the "center of mass" of the eigenvector components for six categories denoted by $c$ as follows:

$$
\left\langle V^{(n)}\right\rangle_{c}=\frac{1}{L_{c}} \sum_{\alpha \in c} V_{\alpha}^{(n)}
$$


where $L_{c}$ are the number of micro prices in the category $c$. The absolute value and the angle of the mean of the above are,

$$
\bar{r}_{n, c}:=\left|\left\langle V^{(n)}\right\rangle_{c}\right|, \quad \bar{\theta}_{n, c}:=\frac{1}{\pi} \arg \left(\left\langle V^{(n)}\right\rangle_{c}\right)(\in[-1,1]) .
$$

Note that the angle or phase represents leads and lags of prices. To study the leads and lags of the micro prices, we calculate the mean square width of the phases, weighted by the radius. To do so, we first define phase difference between the phase of the center of mass and that of the micro price $\alpha$ as

$$
\delta \theta_{n, \alpha}:=\frac{1}{\pi} \arg \left(V_{\alpha}^{(n)}\right)-\bar{\theta}_{n, c}(\in[-1,1]) .
$$

We then define the average of the phase difference and the mean square width of the phase difference, choosing the weights to be the radius $r_{n, \alpha}:=\left|V_{\alpha}^{(n)}\right|$. The average phase $\bar{\theta}_{n, c}$ shows leads (minus) and lags (plus) while the phase spread indicates the variance of leads and lags within a respective group 9 We finally define our phase spread as follows, with correction due to this difference:

$$
\Delta \theta_{n, c}:=\sqrt{\left[\sum_{\alpha \in c} \delta\left(\theta_{n, \alpha}-\delta \bar{\theta}_{n, c}\right)^{2} r_{n, \alpha}\right]\left[\sum_{\alpha \in c} r_{n, \alpha}\right]},
$$

The results are shown in Table 2, The absolute value measures the size of the impact of respective micro price component in the 1st eigenvector on the "true core" aggregate price defined shortly by Eq. 44. The absolute value of CPI is larger than that of DCGPI which is, in turn, larger than that of IPI. Also, the prices of services have the largest absolute value. These results suggest that the 1st eigenvector mainly represents the factors which drive domestic prices.

Fig 31 is for the 2nd, the $3 \mathrm{rd}$, and the 4th eigenvector components in their complex planes in the similar manner. The respective summary measures are listed in Table 3. The absolute values of IPI and oil-related prices are by far the largest for the 2 nd eigenvector. This suggests that the 2 nd eigenvector mainly represents the prices of imported goods such as oil price.

While the absolute value $\bar{r}_{n, c}$ measures the size of the impact of the $n$-th eigenmodes, the phase $\bar{\theta}_{n, c}$ gives lead/lag relationships. The average phase of the 1st eigenvector $\bar{\theta}_{1, c}$ in Table 2 shows that while the first eigenmode basically represents the factors which drive domestic prices, in terms of timing, import prices leads CPI

\footnotetext{
${ }^{9}$ This, however, bring in a difference in a average phase position: Since $\bar{\theta}_{n, c}$ is determined as the phase of the center of mass as in the above, it is a nonlinear average of the phase of the micro prices. Therefore, the mean of the $\delta \theta_{n, \alpha}$ (with any weight) differs from zero in general. In reality, the difference with weight equal to the radius,

$$
\delta \bar{\theta}_{n, c}:=\sqrt{\left[\sum_{\alpha \in c} \delta \theta_{n, \alpha} r_{n, \alpha}\right]\left[\sum_{\alpha \in c} r_{n, \alpha}\right]},
$$

turns out to be very small, i.e. in the range of $[-0.031,0.031]$ for $n=1 \sim 4$ and all $c$.
} 


\begin{tabular}{l|r|r|r}
\hline \multicolumn{1}{c|}{$c$} & $\bar{r}_{1, c}$ & $\bar{\theta}_{1, c}$ & $\Delta \theta_{1, c}$ \\
\hline IPI & 0.019 & -0.539 & 0.173 \\
DCGPI & 0.024 & -0.073 & 0.222 \\
CPI & 0.031 & 0.027 & 0.176 \\
\hline Oil-related & 0.024 & -0.252 & 0.188 \\
Services & 0.046 & 0.038 & 0.094 \\
Construction & 0.028 & -0.097 & 0.223 \\
\hline
\end{tabular}

Table 2. The Average Values for the 1st Eigenvector.

\begin{tabular}{l|r|r|r||r|r|r||r|r|r}
\hline \multicolumn{1}{c|}{$c$} & $\bar{r}_{2, c}$ & $\bar{\theta}_{2, c}$ & $\Delta \theta_{2, c}$ & $\bar{r}_{3, c}$ & $\bar{\theta}_{3, c}$ & $\Delta \theta_{3, c}$ & $\bar{r}_{4, c}$ & $\bar{\theta}_{4, c}$ & $\Delta \theta_{4, c}$ \\
\hline IPI & 0.039 & 0.044 & 0.111 & 0.014 & -0.269 & 0.165 & 0.023 & 0.071 & 0.149 \\
DCGPI & 0.006 & 0.193 & 0.454 & 0.005 & -0.368 & 0.452 & 0.005 & -0.391 & 0.453 \\
CPI & 0.009 & -0.293 & 0.0360 & 0.004 & 0.377 & 0.508 & 0.004 & 0.915 & 0.474 \\
\hline Oil-related & 0.027 & 0.294 & 0.226 & 0.008 & -0.473 & 0.324 & 0.010 & -0.347 & 0.353 \\
Service & 0.018 & -0.252 & 0.215 & 0.012 & 0.949 & 0.364 & 0.012 & 0.655 & 0.348 \\
Construction & 0.010 & 0.235 & 0.40 & 0.012 & -0.424 & 0.351 & 0.010 & -0.244 & 0.352 \\
\hline
\end{tabular}

Table 3. The Average Values for the 2nd to the 4th Eigenvectors.

and service prices. Likewise, while the size of the impact of the second eigenvector $\bar{r}_{2, c}$ by far the largest for IPI, in terms of timing, CPI and service prices (minus sign) lead DCGPI and IPI (plus sign). Tables 2 and 3 also show the variance of timings in each group $\delta \bar{\theta}_{n, c}(n=1,2,3,4)$ for the first to fourth eigenvectors. Though the variances differ, there are, on the whole, significant variances within each group.

The results of the phases suggest that inertia arising from input/output relationships in the production of goods and services is more important than expectations in the determination of prices because the standard assumption of rational expectations based on macro information common to all the firms does not generate systemic leads and lags for well-defined groups of prices. In contrast, inertia arising from input/output relationships in the production of goods and services naturally generate such leads and lags. For example, a rise of oil price would first affect import prices, then prices of intermediate goods (DCGPI), and finally prices of consumption goods and services (Table 3). The comparison of Tables 2 and 3 shows that leads and lags indicated by $\bar{\theta}_{n, c}$ differ for different eigenvectors. Different timings of changes of individual prices mean that inertia of the aggregate price arises mainly from interactions of individual prices through input/output relationships in production and rivalry in the market. This is consistent with the result we obtained in the analysis of autocorrelations of micro prices in Section III 

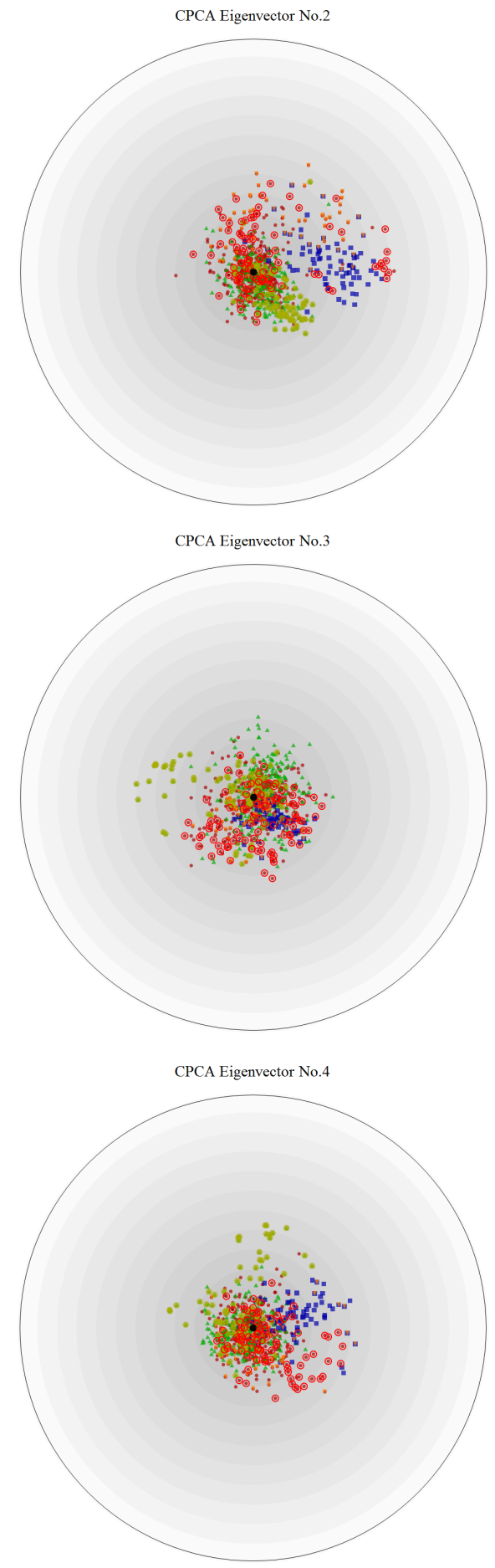

Figure 31. The 2nd to the 4 th eigenvector components in their complex plane. The components of the $n$-th eigenvector are multiplied by a factor $\sqrt{\lambda^{(n)} / \lambda^{(1)}}$, as their contribution is proportional to it as seen in Eq. (33). 


\section{"True Core" Aggregate Price Index}

Given the rate of change of the aggregate price index defined by Eq. (14), we obtain the following expression for the "systemic" change of the aggregate price index by using the mode-signal $a^{(n)}(t)$ defined in Eq. 32 ):

$$
\begin{aligned}
{\left[\frac{\Delta P(t)}{P(t)}\right]^{(\mathrm{tc})} } & :=c \sum_{\alpha=1}^{N} g_{\alpha}\left\langle r_{\alpha}\right\rangle_{t} \frac{p_{\alpha}(t)}{P(t)}+c \Re\left[\sum_{n=1}^{N_{\mathrm{s}}} q^{(n)}(t) a^{(n)}(t)\right], \\
q^{(n)}(t) & :=\sum_{\alpha=1}^{N} g_{\alpha} \frac{p_{\alpha}(t)}{P(t)} V_{\alpha}^{(n)},
\end{aligned}
$$

where $N_{\mathrm{s}}=26$ is the number of significant eigenmodes as before. It is extremely important to note that to understand deflation/inflation, we need to explore the right-hand side of Eq.44 rather than $\Delta P / P$ itself. $[\Delta P / P]^{*}$ defined by the righthand side of Eq. (44) represents the "systemic" part of the aggregate price, a kind of "true core" price. The comparison between the true core CPI and the published CPI is given in Fig. 32 .

In the next section, we will explore with which macro variables our "true core" price defined by Eq. (44) is significantly correlated.

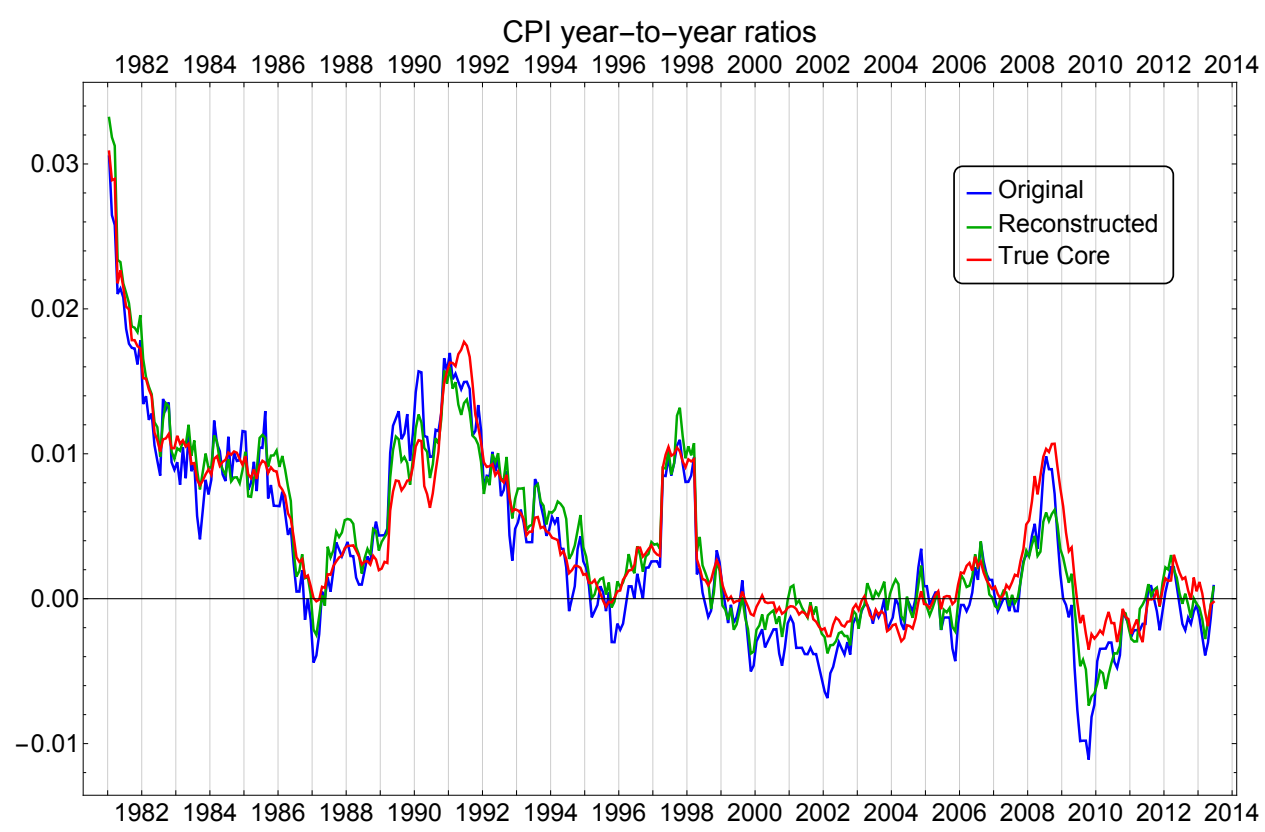

Figure 32. Plot of the year-to-year rate of change of the original CPI (blue), the reconstructed CPI (green), and the "True core" CPI (red). 


\section{Correlation between Mode-signals and Macroeconomic Indices}

For macroeconomics and monetary policy, we are primarily interested in the behavior of the aggregate price index such as CPI. The standard method of regressing the changes of the aggregate price index on various macro-variables aims to answer this question, of course. However, the traditional analysis of the NKPC based on macro data has its clear limitations (Mavroeidis et al., 2014). Meanwhile, the empirical studies of micro prices have amply demonstrated that the aggregate price index which is nothing but the weighted average of individual prices defined by Eq.(12) contains much micro noises. For the purpose of extracting "systemic" movements of the aggregate price index from individual prices, we have constructed eigenmodes based on the correlation matrix of the complexified rates of change of individual prices. This analysis leads us to a kind of "true core" aggregate price defined by Eq.(44). In this section we explore which macro variables these significant eigenmodes represent by examining the correlations of these eigenmodes and macro variables.

The macroeconomic indices used in our study are the followings:

1. Wage index: seasonally adjusted wage index based on contractual cash earnings for establishments with 30 employees or more (source: Monthly Labor Survey; Ministry of Health, Labor and Welfare)

2. Overtime hours worked: including morning work, overtime work, or work on a day off (source: Monthly Labor Survey; Ministry of Health, Labor and Welfare)

3. Unemployment rate: seasonally adjusted (X-12-ARIMA) (source: Labor Force Survey; Ministry of Internal Affairs and Communications)

4. Building Starts: All dwellings, total floor area, seasonally adjusted by Decomp (The Institute of Statistical Mathematics, 2014) with period 12. (source: Ministry of Land, Infrastructure, Transport and Tourism)

5. Monetary base (base money): The sum of banknotes in circulation, coins in circulation, current account deposits held by financial institution at the Bank of Japan; seasonally adjusted (X-12-ARIMA), and average amounts outstanding (source: Bank of Japan)

6. Money stock M2: The quantity of money held by money holders (corporations, households, and local governments including municipal enterprises); M2 is the sum of currency in circulation and deposits; the money issuers are the Bank of Japan, domestically licensed banks (excluding the Japan Post Bank), foreign banks in Japan, Shinkin Central Bank, Shinkin banks, the Norinchukin Bank, and the Shoko Chukin Bank (source: Bank of Japan)

7. Exchange rate (Yen/US Dollar): spot rate at 17:00 in JST, Tokyo market; average in the month (source: Bank of Japan) 
8. Crude oil (petroleum) price index: simple average of three spot prices (Dated Brent, West Texas Intermediate, and the Dubai Fateh, USD, 2005=100) (source: IMF Primary Commodity Prices)

The time period covers exactly the same period for the data of micro prices. All these macro indices except Building starts, Money stock M2, Exchange rate, and Crude oil price index are seasonally adjusted. We use the logarithmic monthly rate of change (see Eq.13) ) of these indices, except for the money stock M2, for which only year-to-year change is available. All these time series of macro price except for the money stock M2 are found to be stationary by the Dicky-Fuller test and the Phillip-Perron tests. For this reason, we also study the logarithmic monthly rate of change of the (year-to-year change of) money stock M2, which we have found to be stationary, and we give this variable a number $\mathbf{6 a}$.

Fig 33 is the plot of all these variables, where all but "6. Money stock M2" are the original value (not the rate of change). "6. Money stock M2" is available only as the year-to-year ratio and is plotted here as the logarithmic rate of change. In examining the correlation of these macro variables with mode signals, we explicitly take into account leads and lags. For this purpose, we complexify these time-series variables by using the method explained in Section[IV]A and denote its standardized (with mean $=0$ and standard deviation $=1$ ) time-series by $\widetilde{M}_{j}(t)$.

In order to investigate the correlation between these macro economic variables and the factors which drive systemic changes of individual prices, namely, modesignals $a^{(n)}(t)$, we calculate the following correlation coefficient:

$$
\mathcal{A}_{j, n}:=\frac{1}{T} \sum_{t=1}^{T} \widetilde{M}_{j}^{*}(t) a^{(n)}(t)
$$

where the index $j$ runs from 1 to 7 for seven macro variable and the index $n$ runs from 1 to 26 for the 26 mode-signals $a^{(n)}(t)$ (defined in Eq. $\left.\sqrt{32}\right)$ ) that represent systemic co-movements of the individual prices of goods and services. Note that because the mode-signals satisfy 33 we have normalized it by dividing by $\sqrt{\lambda^{(n)}}$ so that $\left|\mathcal{A}_{j, n}\right| \in\left[0,2 \sqrt{\lambda^{(n)}}\right]$.

To determine whether the resulting value of $\mathcal{A}_{j, n}$ implies significant correlation between the macro index $j$ and the mode-signal $n$, we utilize the RRS method reviewed in subsection III-IV] To be concrete, we calculate the distribution of the time-shifted correlation

$$
\mathcal{A}_{j, n}^{(\mathrm{RRS})}(\tau):=\frac{1}{T \sqrt{\lambda^{(n)}}} \sum_{t=1}^{T} \widetilde{M}_{j}^{*}(t) a^{(n)}(\operatorname{Mod}(t+\tau, T)+1),
$$

for $\tau=1,2, \cdots, T$ and compare the distribution of the strength of correlations, namely their absolute values, to the absolute value of $\mathcal{A}_{j, n}$.

The results are shown in Fig, 34. In the figure, the absolute values $\left|\mathcal{A}_{j, 1}\right|$ of the first mode-signal are shown by thick bars for seven macro variables from top to bottom. The black dot shows the median of the distribution of $\left|\mathcal{A}_{j, 1}^{(\mathrm{RRS})}(\tau)\right|$, the 
dashed bars the " $1 \sigma$ range", which is the range where $68 \%$ of the RRS results are contained, the solid bars the " $2 \sigma$ range", $95 \% 10$

From Figures 34 and 35 , we draw the following conclusions.

1. The first eigenmode has significant correlations with overtime hours worked, the unemployment rate and the exchange rate.

2. The exchange rate is a very significantly correlated with the 2 nd and the 4 th mode-signals.

3. Neither monetary base nor money stock has significant correlation with any of significant modes.

In Section [V] we found that the absolute value of the first eigenvector for CPI and services is much larger than that for prices of imported and oil-related goods. The result suggests that the first eigenmode represents the factors which drive domestic prices. It is consistent with the finding in the present analysis that the first eigenmode has significant correlations with overtime hours worked and the unemployment rate.

The present analysis demonstrates that systemic movements of micro prices are not correlated with money. In standard macroeconomics models, money is the most important macro variable which affects prices by way of expectations. Our results casts a serious doubt on this standard framework.

\footnotetext{
${ }^{10}$ Note that the RRS result does not obey normal distribution. These ranges are obtained by excluding $16 \%$ largest and $16 \%$ smallest values of the values obtained by random rotation for the " $1 \sigma$ " range and similarly for " $2 \sigma$ " range.
} 

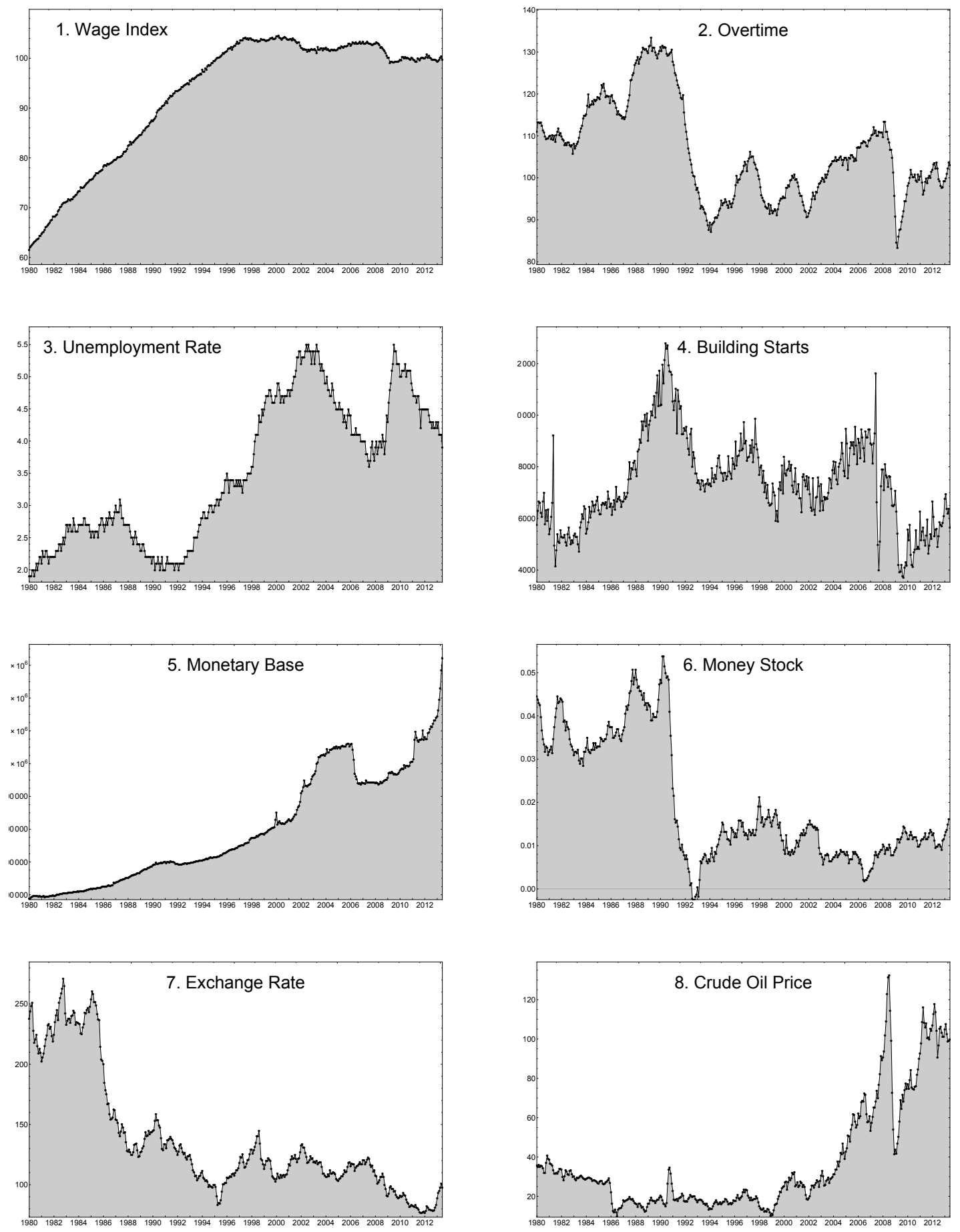

Figure 33. The eight macroeconomic indices that we study. 


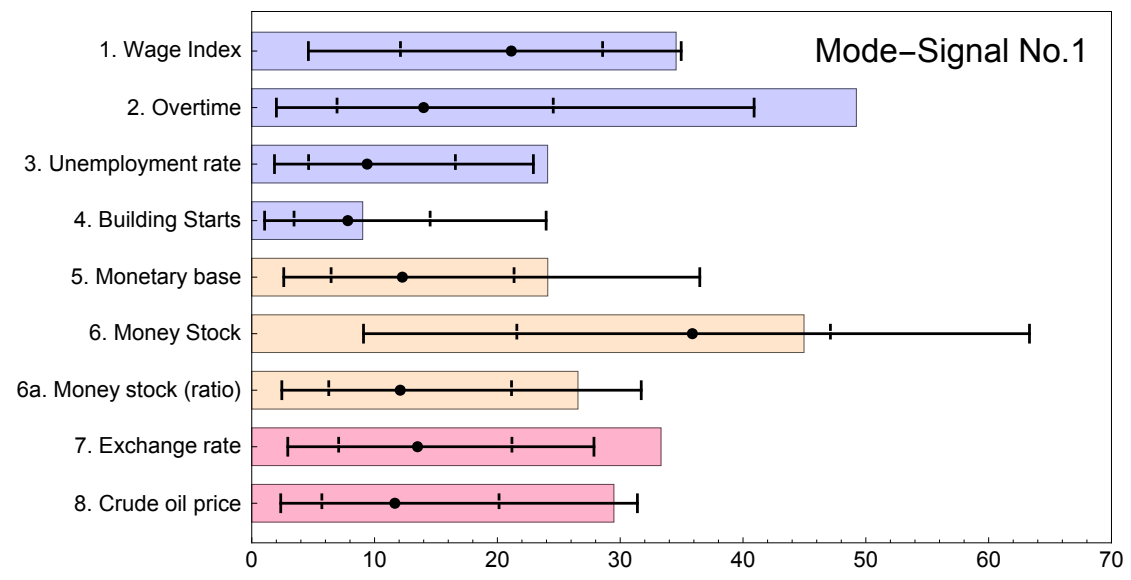

Figure 34. The absolute values of the correlation coefficient $\mathcal{A}_{j, 1}$ with their RRS ranges.
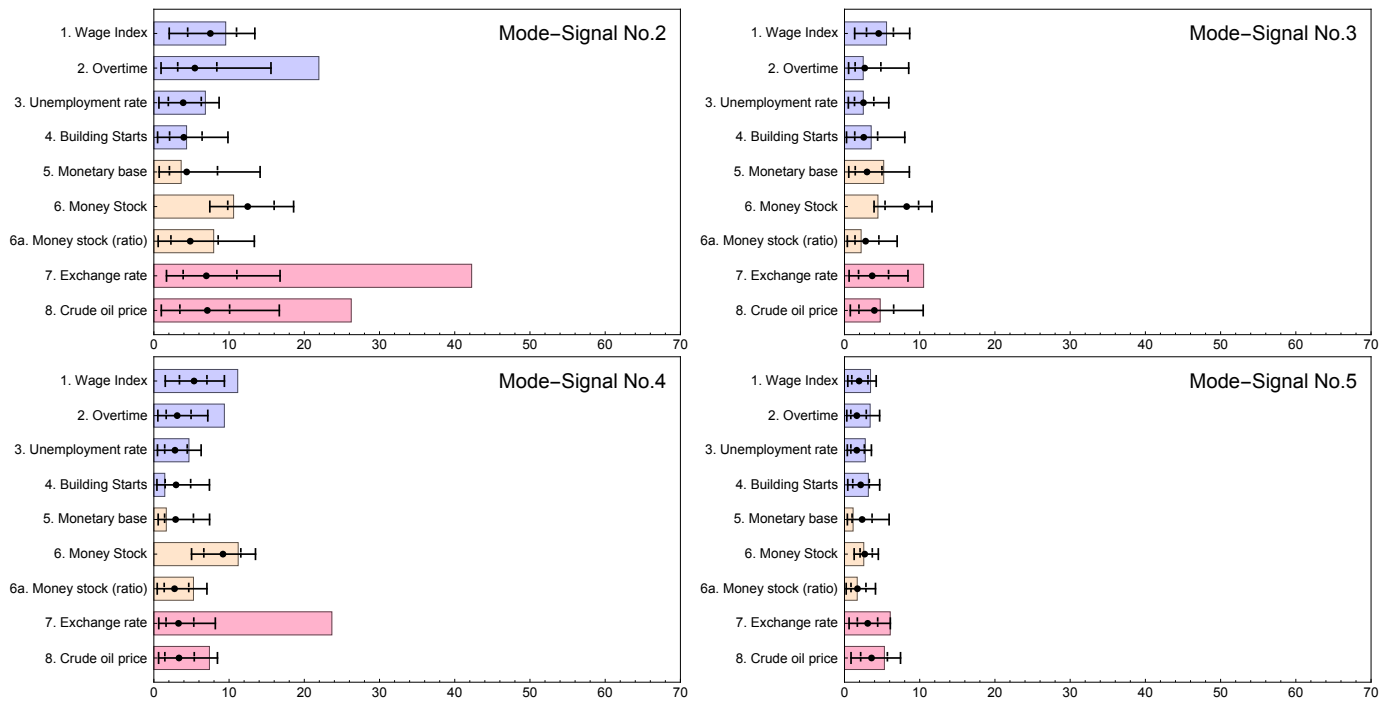

Figure 35. The absolute values of the correlation coefficient $\left|\mathcal{A}_{j, n}\right|$ for $n=2-5$ with their RRS ranges. 


\section{Concluding Remarks}

The traditional analysis of the New Keynesian Phillips curve based on macro data has its clear limitations in the exploration of dynamics of aggregate price, namely deflation/inflation (Mavroeidis et al., 2014). Meanwhile, empirical research on individual prices in the past decade has uncovered the details of micro price dynamics. It has demonstrated that there is a considerable cross-sectional heterogeneity in the frequency and/or the hazard rate of price change across goods and services (Carvalho (2006), Klenow and Malin (2011)). The information is useful for understanding industrial organization of particular market. However, it provides only a limited information on deflation/inflation precisely because understanding deflation/inflation amounts, after all, to understanding changes in the behavior of the aggregate price over time. The existing literature focuses on cross-sectional distribution of micro price changes, and assumes that the distribution is given and time-invariant. We note that micro-optimization exercise results in a particular pattern of price setting which is time-invariant.

Our analysis casts serious doubt on the standard framework of macroeconomics and monetary policy. The problems are two-fold: The first is the role of expectations in the determination of aggregate price. The second perhaps more fundamental problem is the relation between price and real output.

Section II of the paper demonstrated that changes of micro prices which produce deflation/inflation are time-varying. The existing literature finds that the frequency of price change rises under high inflation. This finding, however, has little relevancy to understanding deflation/inflation under "normal" situations. Gordon (2011), in fact, points out that different models must apply to big inflations on one hand, and "normal" situations such as the postwar experiences of the advanced economies on the other. He then argues that forward-looking model with emphasis on role of expectations applies to big inflations whereas model with persistence and inertia to "normal" situations. In normal situations, the frequency of price change provides only a limited information. Our analyses support Gordon (2011)'s assertion in that persistence and inertia is more important than expectations in the determination of aggregate price index. First, the analysis of autocorrelations in Section III demonstrated the significance of cross-autocorrelations of micro prices in the aggregate price dynamics. The standard assumption of rational expectations does not generate such cross-autocorrelations of micro prices whereas they naturally arise from input/output relationships in production of goods and services (Gordon, 2011, pp.32). Secondly, the analysis in Section IV showed that there exists a significant dispersion in the timing of changes of micro prices. The standard assumption of rational expectations based on macro information common to all the firms does not generate systemic leads and lags for well-defined groups of prices. In contrast, inertia arising from input/output relationships in the production of goods and services naturally generate such leads and lags. We can recall that the cost-based mark-up pricing was once said

to be prevalent (Hall and Hitch (1939), Nordhaus and Godley (1972)). Eichenbaum et al. (2011) using scanner data from a US supermarket chain, also shows that retail 
prices, reference prices excluding temporary sales in particular, tend to change so as to keep the product's mark up over marginal cost at its average level.

The second fundamental problem is the relation between price and real output. The current literature takes it that inflexibility of nominal prices produces fluctuations of real output. It presumes that changes in real economic activity arise largely from fluctuations in nominal aggregate demand which, in turn, are conditioned by money supply. The basic framework is a variant of money demand/supply equation:

$$
M=k P Y \quad(k>0) .
$$

Monetary policy identified as a change in nominal money supply $M$ generates little change in real output $Y$ if nominal price $P$ instantaneously changed in proportion to $M$. In contrast, it entails a change in $Y$ if whatever the reason, $P$ is inflexible. A succinct presentation of this model can be found in Nakamura and Steinsson (2013). Given this framework, the existing literature on micro prices is interested first in how inflexible nominal price actually is, and secondly, in discriminating competing theories which attempt to provide micro-foundations for inflexible nominal price $\overline{(B i l s}$ et al. 2003).

Given the results we obtained in the present paper, the validity of this standard theoretical framework is open to doubt. In the first place, the analysis in Section $\mathrm{V}$ shows that changes in the aggregate price index, namely deflation or inflation, consistent with systemic fluctuations of micro prices are not directly linked to changes in money supply such as M2 and base money; The "true core" aggregate price index defined by Eq. (44) can change independent of changes of money supply. The reason is that except for at the irregular zero interest rate bound, monetary policy is interest rate policy everywhere making money supply endogenous, or even passive as Black (1986, p.539) observes; In terms of Eq. 48), $k$ is not constant but endogenously changes responding to $M$.

There appear two dominant factors (eigenmodes) which produce changes in the "true core" aggregate price. The first eigenmode, namely the most important factor, is significantly correlated with overtime hours worked and unemployment rate. It is extremely important to note that overtime hours worked and the unemployment rate are direct measures of real production or output, not nominal demand.

The result is consistent with the old Phillips curve which says that in booms, both quantities and prices change upward while the converse holds true in recessions. Note that the Phillips curve is not a mere correlation between price and quantity. It is not the case that quantities change because prices do not change. Rather prices change responding positively to changes in quantities. Causality runs from the level of real output to changes in prices. The Phillips curve, a macro equation, emerges from aggregation of heterogeneous markets (Lipsey (1960), Tobin (1972), Okun (1981)). The bottom line is that the aggregate price index rises when the average level of real economic activity as represented by overtime hours worked or the unemployment rate goes up.

As for changes in quantities, the best explanation is given by Tobin (1993): 
"The central Keynesian proposition is not nominal price rigidity but the principle of effective demand (Keynes, 1936, Ch.3). In the absence of instantaneous and complete market clearing, output and employment are frequently constrained by aggregate demand. In these excesssupply regimes, agents' demands are limited by their inability to sell as much as they would like at prevailing prices. Any failure of price adjustments to keep markets cleared opens the door for quantities to determine quantities, for example real national income to determine consumption demand, as described in Keynes' multiplier calculus...

In Keynesian business cycle theory, the shocks generating fluctuations are generally shifts in real aggregate demand for goods and services, notably in capital investment. Keynes would be appalled to see his cycle model described as one in which "fluctuations in output arise largely from fluctuations in nominal aggregate demand" (Ball, Mankiw, and Romer 1988, p.2). The difference is important." (Tobin, 1993)

The best micro-foundation is given by Negishi (1979). The point is that real demand determines real output, and then, real output affects prices.

The second important factor (eigenmode) generating the systemic fluctuations of individual prices is significantly correlated with the exchange rate and crude oil price. In open economy like the Japanese economy, changes in the exchange rate and oil price affect the import prices without lags, and they, in turn, change the costs of energy and materials used in the production of a wide range of goods and services. With lags, many prices follow suit 11 The case study of the Post Plaza Agreement period when the yen sharply appreciated from 240 per dollar to 120 amply demonstrates the present of this mechanism. In fact, Brown and Ozga (1955) studying the long-term data (1870-1950) for the U.K. found that the most important determinant of the British price was terms of trade which was in turn basically determined by prices of raw materials. It is easy to dismiss this finding by saying that price is nominal whereas terms of trade are real. But that is what data tells us. For the Japanese economy, real price of energy and the real exchange rate affect the nominal aggregate price. Gordon (2011) also emphasizes the importance of this factor forgotten in the recent literature on NKPC under the heading of "supply shock".

Deflation and inflation are macroeconomic phenomena. However, we cannot fully understand them by only exploring macro data because the behavior of aggregate price such as CPI depends crucially on interactions of micro prices. On the other hand, systemic comovements of micro prices are, in turn, conditioned strongly by the state of the macroeconomy. All in all, the results we obtained have confirmed that the aggregate price significantly changes, either upward or downward, as the level of real output changes. The correlation between the aggregate price and money, on the

\footnotetext{
${ }^{11}$ Gopinath et al. (2010) find that exchange rates systematically affect import prices for the U.S. as well, but that the elasticity of import prices with respect to changes in exchange rates is rather small, namely that firms adjust prices by only $0.25 \%$ for each $1 \%$ change in the exchange rate.
} 
other hand, is not significant. The major factors affecting the aggregate price other than the level of real economic activity are the exchange rate and the prices of raw materials represented by the price of oil. Japan had suffered from deflation for more than a decade beginning the end of the last century. More recently, Europe faces a threat of deflation. Our analysis suggests that it is difficult to combat deflation only by expanding money.

\section{Acknowledgments}

This work is partially supported by Grant-in-Aid for Scientific Research (KAKENHI) Grant Numbers 24243027 and 25282094 by JSPS, and the European Community Seventh Framework Programme (FP7/2007-2013) under Socio-economic Sciences and Humanities, grant agreement no. 255987 (FOC-II) and "FOC-INCO" 297149.

The authors would like to thank Professors Yuichi Ikeda, Wataru Souma, Kenichi Ueda, Tsutomu Watanabe, and Yoshihiro Yajima for their helpful discussions, and K. Itoh and N. Shinozaki (both at NHK -Japan Broadcasting Corporation) for their assistance in data acquisition. We are also grateful to the participants of seminars at the Bank of Japan, University of Tokyo, and the Discussion Paper Seminar at RIETI for their useful comments. 


\section{Appendix A. CPCA for sine and cosine curves}

In order to demonstrate the power of CPCA used in the present paper, let us take the following two time-series:

$$
r_{1}(t)=\sin \left(\frac{\pi t}{23}\right), \quad r_{2}(t)=\cos \left(\frac{\pi t}{23}\right)
$$

for $t=0,1,2, \cdots, 99$, which is plotted in Fig 36 .

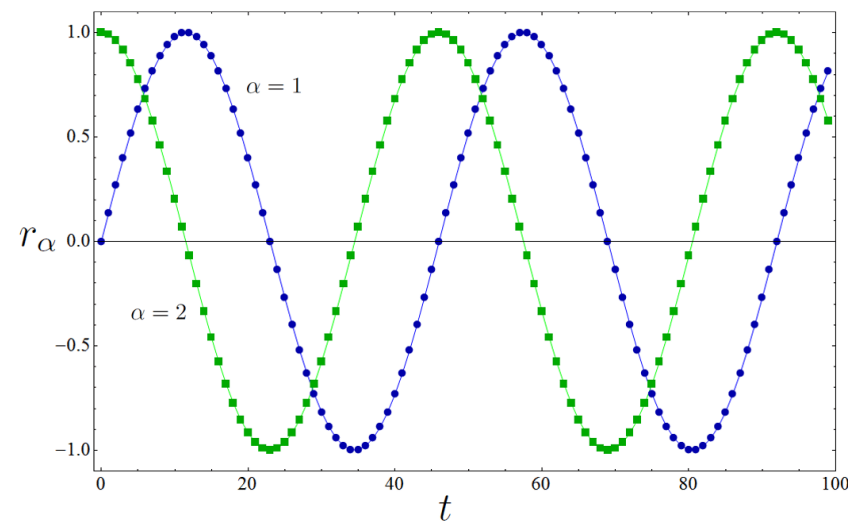

Figure 36. The sample time-series of sin and cos defined in Eq.49.

The PCA correlation matrix is the following:

$$
\boldsymbol{C}=\left(\begin{array}{cc}
1 & 0.049 \\
0.049 & 1
\end{array}\right)
$$

which fails to detect correlation with time-lag between these two time-series.

When complexified, these time-series behaves as shown in Fig. 37 Note that the period of these sinusoidal curves is equal to 46, which is not a divider of the whole time range $T=100$. Therefore, these time-series do not have just one Fourier component (see Eq. 15 ), which explains the fact that the beginning part, say, $t \lesssim 10$ and the ending part, $t \gtrsim 90$. Nonetheless, the overall rotation of the time-series in the complex plane is remarkably reproduced accurately, except for these edge regions.

The CPCA correlation matrix defined in Eq. (23) is now as follows:

$$
\widetilde{\boldsymbol{C}}=\left(\begin{array}{cc}
1 & 0.981 e^{0.484 \pi i} \\
0.981 e^{-0.484 \pi i} & 1
\end{array}\right)
$$

And the eigenvalues and eigenvectors are the following:

$$
\begin{array}{ll}
\lambda^{(1)}=1.982, & \boldsymbol{V}^{(1)}=\frac{1}{\sqrt{2}}\left(\begin{array}{c}
1 \\
e^{-0.484 \pi}
\end{array}\right), \\
\lambda^{(2)}=0.019, \quad \boldsymbol{V}^{(2)}=\frac{1}{\sqrt{2}}\left(\begin{array}{c}
1 \\
e^{+0.484 \pi}
\end{array}\right) .
\end{array}
$$




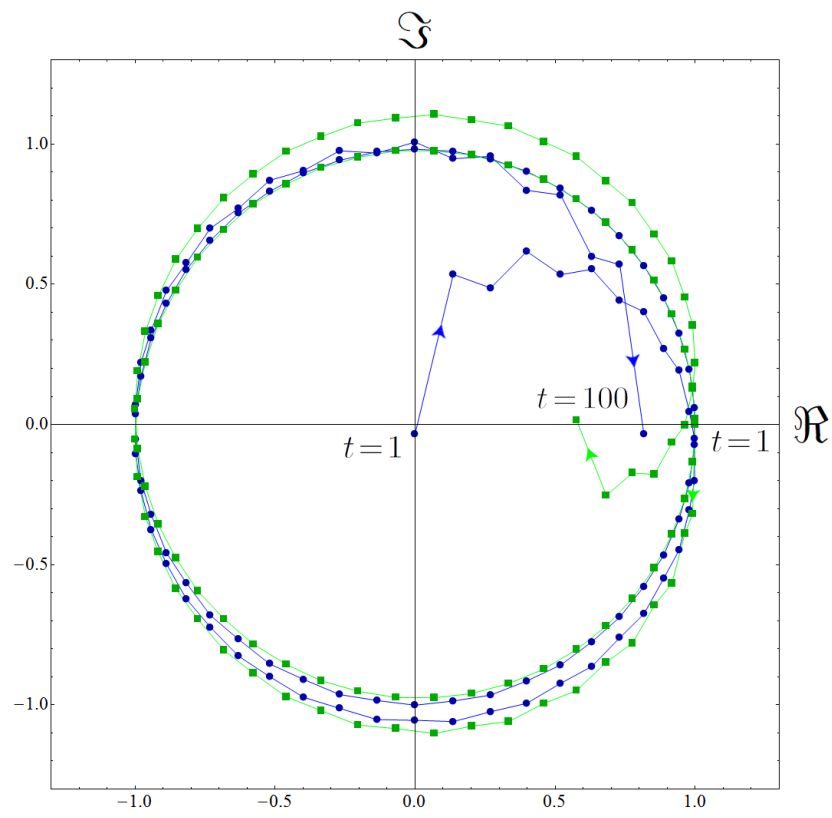

Figure 37. The time-series $\widetilde{r}_{1,2}(t)$ complexified as defined in Eq. (18).

The phase $\delta_{12}=0.48 \pi$ shows that the time-series $r_{1}$ lags behind $r_{1}$ by $0.484 \pi$ (see Fig 17), which is very close to the actual value, $0.5 \pi$. Furthermore, its absolute value $\left|\widetilde{C}_{12}\right|=0.981$ implies that the correlation with this time-lag is very strong, or almost perfect, which is the desired result. The eigenmode 1 is indeed the comovement of the sine and cosine with time-lag.

This demonstrates the strength of CPCA for detecting correlations with lead/lag. 


\section{References}

Arai, Y. and H. Iyetomi, "Complex Principal Component Analysis of Dynamic Correlations in Financial Markets," Intelligent Decision Technologies, Frontiers in Artificial Intelligence and Applications, 2013, 255, 111-119.

Bank of Japan, "Outline of Statistics and Statistical Release Schedule," 2014. https://www.boj.or.jp/en/statistics/outline/exp/pi/excgpi02.htm/.

Basawa, I. V. and B. L. S. Prakasa Rao, Statistical Inference for Stochastic Processes, Academic Press, 1980.

Bils, M., P. J. Klenow, and O. Kryvtsov, "Sticky Prices and Monetary Policy Shocks," Federal Reserve Bank of Minneapolis Quarterly Review, 2003, 27 (1), $2-9$.

Bils, Mark and Peter J Klenow, "Some Evidence on the Importance of Sticky Prices," Journal of Political Economy, October 2004, 112, 947-85.

Black, Fischer, "Noise," The journal of finance, 1986, 41 (3), 529-543.

Brown, E. H. Phelps and S. Andrew Ozga, "Economic growth and the price level," The Economic Journal, 1955, pp. 1-18.

Buja, A. and N. Eyuboglu, "Remarks on parallel analysis," Multivariate Behavioral Research, 1992, 27, 509-540.

Calvo, Guillermo A, "Staggered prices in a utility-maximizing framework," Journal of monetary Economics, 1983, 12 (3), 383-398.

Carvalho, Carlos, "Heterogeneity in price stickiness and the real effects of monetary shocks," Frontiers in Macroeconomics, 2006, 2 (1).

Dickey, David A and Wayne A Fuller, "Distribution of the estimators for autoregressive time series with a unit root," Journal of the American statistical association, 1979, 74 (366a), 427-431.

Eichenbaum, M., N. Jaimovich, and S. Rebelo, "Reference Prices, Costs, and Nominal Rigidities," American Economic Review, 2011, 101, 234-262.

Franklin, Scott B., David J. Gibson, Philip A. Robertson, John T. Pohlmann, and James S. Fralish, "Parallel Analysis: a method for determining significant principal components," Journal of Vegetation Science, 1995, 6, 99-106.

Golosov, Mikhail and Robert E Lucas, "Menu costs and Phillips curves," Journal of Political Economy, April 2007, 115, 171-199.

Gopinath, Gita, Oleg Itskhoki, and Roberto Rigobon, "Currency choice and exchange rate pass-through," American Economic Review, 2010, 100 (1), 304-336. 
Gordon, Robert J, "The history of the Phillips curve: consensus and bifurcation," Economica, 2011, 78 (309), 10-50.

Granger, Clive William John and Michio Hatanaka, Spectral analysis of economic time series., Princeton Univ. Press., 1964.

Hall, Robert L and Charles J Hitch, "Price theory and business behaviour," Oxford economic papers, 1939, 2, 12-45.

Horn, J.L., "A rationale and test for the number of factors in factor analysis," Psychometrica, 1965, 30, 179-185.

Iyetomi, Hiroshi, Yasuhiro Nakayama, Hiroshi Yoshikawa, Hideaki Aoyama, Yoshi Fujiwara, Yuichi Ikeda, and Wataru Souma, "What causes business cycles? Analysis of the Japanese industrial production data," Journal of the Japanese and International Economies, 2011, 25 (3), 246-272.

Klenow, Peter J and Benjamin A Malin, "Microeconomic evidence on pricesetting," in Benjamin H. Friedman and Michael Woodford, eds., Handbook of Monetary Economics, Vol. 3A, North-Holland, 2011, chapter 6.

Lipsey, Richard G, "The relation between unemployment and the rate of change of money wage rates in the United Kingdom, 1862-1957: A further analysis," Economica, 1960, pp. 1-31.

Mankiw, N Gregory, "Small menu costs and large business cycles: A macroeconomic model of monopoly," The Quarterly Journal of Economics, 1985, pp. 529537 .

Marčenko, V. A. and L. A. Pastur, "Distribution of eigenvalues for some sets of random matrices," Mathematics of the USSR-Sbornik, 1967, 1 (4), 457-483.

Mavroeidis, Sophocles, Mikkel Plagborg-Møller, and James H Stock, "Empirical evidence on inflation expectations in the New Keynesian Phillips Curve," Journal of Economic Literature, 2014, 52 (1), 124-188.

Midrigan, Virgiliu, "Menu costs, multiproduct firms, and aggregate fluctuations," Econometrica, 2011, 79 (4), 1139-1180.

Nakamura, Emi and Jón Steinsson, "Price rigidity: Microeconomic evidence and macroeconomic implications," National Bureau of Economic Research Working Paper Series, 2013, 18705.

Negishi, T., Microeconomic Foundation of Keynesian Macroeconomics, NorthHolland Publishing, 1979.

Nordhaus, William D and Wynne Godley, "Pricing in the trade cycle," The Economic Journal, 1972, pp. 853-882. 
Okun, Arthur M, Prices and quantities: A macroeconomic analysis, Brookings Institution Press, 1981.

Phillips, Peter CB and Pierre Perron, "Testing for a unit root in time series regression," Biometrika, 1988, 75 (2), 335-346.

Statistics Bureau, "Consumer Price Index," 2014. http://www.stat.go.jp/ english/data/cpi/index.htm.

Statistics Japan, "Q\&A on the Consumer Price Index (in Japanese)," 2014. http: //www.stat.go.jp/data/cpi/4-1.htm.

The Institute of Statistical Mathematics, "Web Decomp - Seasonal Adjustment \& Time Series Analysis," 2014. http://ssnt.ism.ac.jp/inets/inets_ eng.html.

Tobin, James, "Inflation and unemployment," American economic review, 1972, $62(1), 1-18$.

—, "Price Flexibility and Output Stability: An Old Keynesian View," Journal of Economic Perspectives, 1993, 7, 45-65.

Vodenska, Irena, Hideaki Aoyama, Yoshi Fujiwara, Hiroshi Iyetomi, Yuta Arai, and H. Gene Stanley, "Interdependencies and causality in coupled fnancial networks," Available at SSRN 2477242, 2014.

Zwick, W.R. and W.F. Velicer, "Comparison of five rules for determining the number of components to retain," Psychological Bulletin, 1986, 99, 432-442. 